\title{
Izu Vaisman
}

Variétés riemanniennes feuilletées

Czechoslovak Mathematical Journal, Vol. 21 (1971), No. 1, 46-75

Persistent URL: http://dml.cz/dmlcz/101003

\section{Terms of use:}

(C) Institute of Mathematics AS CR, 1971

Institute of Mathematics of the Czech Academy of Sciences provides access to digitized documents strictly for personal use. Each copy of any part of this document must contain these Terms of use.

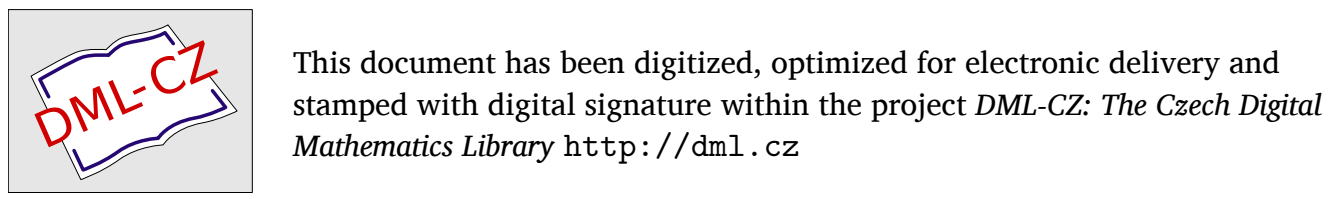




\section{VARIÉTÉS RIEMANNIENNES FEUILLETÉES}

IzU VaISman, Jassy

(Reçu le 22 septembre 1969)

Les variétés feuilletées ont été étudiées par divers auteurs, notamment par EHREsmann, Reeb, Haefliger, Reinhart, Hermann, Palais etc., surtout au point de vue topologique. Des considérations de géométrie différentielle sur ces variétés se trouvent chez Reinhart, Hermann, Deheuvels, Molino, etc. Ici, nous nous proposons d'étudier du point de vue de la géometrie différentielle les variétés feuilletées douées encore d'une métrique riemannienne. Une part des résultats que nous exposons ont été annoncés dans [13].

\section{VARIÉTÉS RIEMANNIENNES PRESQUE-FEUILLETÉES}

1.1. Soit $V^{n+m}$ une variété différentiable, paracompacte à $n+m$ dimensions. La différentiabilité sera toujours comprise dans le sens $C^{\infty}$ et celle-ci sera la classe de toutes les fonctions et champs envisagés.

On définit sur $V^{n+m}$ une structure riemannienne presque feuilletée, en se donnant une métrique riemannienne positivement définie $g$ et une distribution $m$-dimensionelle régulière $\mathscr{D} . V^{n+m}$ douée de cette structure sera nommée variété riemannienne presque feuilletée de codimension $n$ (v.r.p.f.) et alors on a, dans chaque point $M \in V^{n+m}$ un sous-espace vectoriel $m$-dimensionnel $D_{M}^{m}$ de l'espace tangent $T_{M}^{n+m}$, celui-ci dépendant différentiablement du point $M$. Rappelons que la géométrie locale d'une pareille distribution a été étudiée par R. Miron et D. PAPUC [6].

Sur la v.r.p.f. $V^{n+m}$, on a encore la distribution $\mathscr{D}^{\perp}$, définie par les $n$-plans $D_{M}^{\perp}$ orthogonaux à $D_{M}^{m}$; elle sera nommée la distribution complémentaire de $\mathscr{D}$ et on a

$$
T_{M}=D_{M} \oplus D_{M}^{\perp},
$$

donc $V^{n+m}$ possède une structure presque-produit canonique $\mathscr{P}[7]$.

On dira aussi que $\mathscr{D}$ est la distribution structurelle et $\mathscr{D}^{\perp}$ la distribution transversale de la v.r.p.f. 
Donnons maintenant l'expression locale de la structure considérée. Nous convenons de noter $i, j, \ldots=1, \ldots, n+m ; a, b, \ldots=1, \ldots, n ; u, v, \ldots=n+1, \ldots$ $\ldots, n+m$.

La structure de v.r.p.f. peut être considérée définie par un recouvrement ouvert $\mathscr{U}=\left\{U_{\alpha}\right\}$ de $V^{n+m}$ et par la définition, dans chaque $U_{\alpha}$, de deux systèmes de formes de Pfaff

$$
\omega_{\alpha}^{a}, \omega_{\alpha}^{u},
$$

linéairement indépendantes et tels que, dans $U_{\alpha} \cap U_{\beta}$, on ait

$$
\omega_{\beta}^{a}=p_{b}^{a} \omega_{\alpha}^{b}, \quad \omega_{\beta}^{u}=q_{v}^{u} \omega_{\alpha}^{v},
$$

où $\left(p_{b}^{a}\right)$ et $\left(q_{v}^{u}\right)$ sont des matrices orthogonales.

Ces formes sont telles que, dans $U_{\alpha}, \mathscr{D}$ soit définie par les équations

$$
\omega_{\alpha}^{a}=0,
$$

$\mathscr{D}^{\perp}$ par les équations

$$
\omega_{\alpha}^{u}=0
$$

et $g$ ait l'expression

$$
\mathrm{d} s^{2}=\sum_{a}\left(\omega_{\alpha}^{a}\right)^{2}+\sum_{u}\left(\omega_{\alpha}^{u}\right)^{2} .
$$

Nous considérerons aussi les bases locales duales aux cobases (1.2)

$$
V_{a}^{\alpha}, V_{u}^{\alpha}
$$

et alors $D_{M}$ est engendré par les vecteurs $V_{u}^{\alpha}$ et $D_{M}^{\perp}$ par $V_{a}^{\alpha}$; les formules de passage de $U_{\alpha}$ à $U_{\beta}$ résultent immédiatement de (1.3).

La considération des bases (1.7) montre que si l'on note

$$
D\left(V^{n+m}\right)=\bigcup_{M \in V^{n+m}} D_{M}, \quad D^{\perp}\left(V^{n+m}\right)=\bigcup_{M \in V^{n+m}} D_{M}^{\perp},
$$

on obtient deux fibrés vectoriels, de base $V^{n+m}$, qui seront nommés respectivement le fibré structurel et le fibré transversal de la v.r.p.f. et dont la somme de Whitney est le fibré tangent de la variété. Dorénavant, nous renoncerons à l'indice $\alpha$ et aussi à l'indice de dimension de la variété.

Les formes (1.2) peuvent servir à exprimer localement les formes différentielles de $V$, qui se présenteront naturellement comme sommes de formes du type $(p, q)$ $(0 \leqq p \leqq n, 0 \leqq q \leqq m)$ :

$$
\varphi=\frac{1}{p ! q !} \varphi_{a_{1} \ldots a_{p} u_{1} \ldots u_{q}} \omega^{a_{1}} \wedge \ldots \wedge \omega^{a_{p}} \wedge \omega^{u_{1}} \wedge \ldots \wedge \omega^{u_{q}},
$$


où $\varphi_{a_{1} \ldots a_{p} u_{1} \ldots u_{q}}$ sont antisymétriques en $a_{1} \ldots a_{p}$ et $u_{1} \ldots u_{q}$ séparément. Le type de la forme (1.9) est invariant en vue des formules de transformation (1.3).

Ainsi, par exemple, on a des formules de la forme suivante

$$
\begin{aligned}
& \mathrm{d} \omega^{a}=\frac{1}{2} \lambda_{b c}^{a} \omega^{b} \wedge \omega^{c}+\lambda_{b u}^{a} \omega^{b} \wedge \omega^{u}+\frac{1}{2} \lambda_{u v}^{a} \omega^{u} \wedge \omega^{v}, \\
& \mathrm{~d} \omega^{u}=\frac{1}{2} \lambda_{a b}^{u} \omega^{a} \wedge \omega^{b}+\lambda_{a v}^{u} \omega^{a} \wedge \omega^{v}+\frac{1}{2} \lambda_{v w}^{u} \omega^{v} \wedge \omega^{w},
\end{aligned}
$$

où les $\lambda$ sont des fonctions sur $U_{\alpha}$.

De (1.10) il suit que la distribution $\mathscr{D}$ est intégrable si et seulement si

$$
\lambda_{u v}^{a}=0
$$

et $\mathscr{Q}^{\perp}$ est intégrable si et seulement si

$$
\lambda_{a b}^{u}=0
$$

1.2 On sait [2] que si $E$ est un fibré vectoriel sur $V$, une connexion de $E$ est un opérateur

$$
D: \Gamma(E) \rightarrow \Gamma\left(T^{*} \otimes E\right),
$$

où $T^{*}$ est le fibré cotangent de $V$ et $\Gamma$ est le foncteur qui associe à chaque fibré vectoriel l'espace de ses sections globales, opérateur satisfaisant aux conditions

$$
D\left(\gamma_{1}+\gamma_{2}\right)=D\left(\gamma_{1}\right)+D\left(\gamma_{2}\right), \quad D(f \gamma)=\mathrm{d} f \otimes \gamma+f D \gamma,
$$

où $\gamma, \gamma_{1}, \gamma_{2} \in \Gamma(E)$ et $f$ est une fonction différentiable sur $V$.

Si maintenant $F$ est un sous-fibré vectoriel de $E$ et si la restriction $\left.D\right|_{\Gamma(F)}$ prend des valeurs dans $\Gamma\left(T^{*} \otimes F\right)$, il est clair que la connexion $D$ induit une connexion du fibré $F$. Classiquement, ceci revient au fait que le transport par parallélisme par rapport à $D$ conserve l'ensemble des fibres de $F$.

Localement, la connexion s'exprime par les équations classiques de connexion d'Elie Cartan [1].

Une première connexion canoniquement associée à la v.r.p.f. $V$ est la connexion de Levi-Civita de la métrique $g$; elle sera nommée la première connexion de $V$. Localement elle est donnée par les équations [1]

$$
D V_{a}=\bar{\omega}_{a}^{b} V_{b}+\bar{\omega}_{a}^{u} V_{u}, \quad D V_{u}=\bar{\omega}_{u}^{a} V_{a}+\bar{\omega}_{u}^{w} V_{w},
$$

où les $\bar{\omega}$ sont des formes de Pfaff pour lesquelles on a

$$
\bar{\omega}_{a}^{b}+\bar{\omega}_{b}^{a}=0, \quad \bar{\omega}_{a}^{u}+\bar{\omega}_{u}^{a}=0, \quad \bar{\omega}_{u}^{w}+\bar{\omega}_{w}^{u}=0 .
$$

Il est classique de trouver l'expression des formes $\bar{\omega}$ à l'aide des coefficients $\lambda$ de (1.10) et en tenant compte des équations de structure

$$
\mathrm{d} \omega^{i}=\omega^{i} \wedge \bar{\omega}_{i}^{j}
$$


Si l'on note

$$
\bar{\omega}_{j}^{i}=\gamma_{j k}^{i} \omega^{k}
$$

et si l'on emploie (1.14), (1.15) et (1.10), on trouve

$$
\gamma_{j k}^{i}=\frac{1}{2}\left(\lambda_{j k}^{i}+\lambda_{k i}^{j}-\lambda_{i j}^{k}\right), \quad\left(\lambda_{j k}^{i}=-\lambda_{k j}^{i}\right) .
$$

Des équations (1.13) il suit que cette connéxion induit une connexion du fibré transversal de $V$ si et seulemenet si

$$
\bar{\omega}_{a}^{u}=0,
$$

c'est-à-dire

$$
\lambda_{a b}^{u}+\lambda_{b u}^{a}-\lambda_{u a}^{b}=0, \quad \lambda_{a v}^{u}+\lambda_{v u}^{a}-\lambda_{u a}^{v}=0 .
$$

Elle induit une connexion du fibré structurel si et seulement si

$$
\bar{\omega}_{u}^{a}=0,
$$

$\mathrm{ou}$

$$
\lambda_{u b}^{a}+\lambda_{b a}^{u}-\lambda_{a u}^{b}=0, \quad \lambda_{u v}^{a}+\lambda_{v a}^{u}-\lambda_{a u}^{v}=0 .
$$

La seconde relation (1.14) montre que ces conditions sont simultanément vérifiées.

Si l'on change le rôle des indices $a$ et $b$, et respectivement $u$ et $v$, dans (1.19) on a

$$
\lambda_{b a}^{u}+\lambda_{b u}^{a}-\lambda_{u a}^{b}=0, \quad \lambda_{a u}^{v}+\lambda_{u v}^{a}-\lambda_{v a}^{u}=0
$$

et la somme de ces relations et celles de (1.19) donne

$$
\lambda_{u v}^{a}=0, \quad \lambda_{a b}^{u}=0,
$$

donc, d'après (1.11) et (1.12), il résulte le

Théorème 1.1. Si la première connexion de la v.r.p.f. Vinduit une connexion dans le fibré structurel (transversal), alors elle induit aussi une connexion dans le fibré transversal (structurel) et les distribution $\mathscr{D}$ et $\mathscr{D}^{\perp}$ sont intégrables.

1.3. Nous introduirons maintenant une seconde connexion sur la v.r.p.f. $V$. Supposons qu'elle a les équations

$$
\begin{aligned}
& D V_{a}=\omega_{a}^{b} V_{b}+\omega_{a}^{u} V_{u}, \\
& D V_{u}=\omega_{u}^{a} V_{a}+\omega_{u}^{w} V_{w},
\end{aligned}
$$

où

$$
\omega_{i}^{j}=\Gamma_{i k}^{j} \omega^{k}
$$


Pour déterminer cette connexion, nous imposerons trois conditions. La première est que la connexion (1.22) induise des connexions dans les fibrés $D(V)$ et $D^{\perp}(V)$, ce qui revient à

$$
\omega_{a}^{u}=\omega_{u}^{a}=0
$$

La seconde condition est que ces connexions induites soient compatibles, pour les courbes tangentes à $\mathscr{D}$, respectivement à $\mathscr{D}^{\perp}$, avec les métriques induites naturellement par $g$ dans $D(V)$ et $D^{\perp}(V)$, ou, ce qui est la même chose, que le transport par parallélisme le long des courbes tangentes à $\mathscr{D}$ et à $\mathscr{D}^{\perp}$, respectivement, conserve la longueur des vecteurs de $\mathscr{D}$ ou $\mathscr{D}^{\perp}$ respectivement.

Ceci revient à

$$
\omega_{a}^{b}+\omega_{b}^{a}=0\left(\operatorname{modulo} \omega^{u}\right), \omega_{u}^{v}+\omega_{v}^{u}=0\left(\operatorname{modulo} \omega^{a}\right) .
$$

Pour imposer la troisième condition, considérons la torsion de notre connexion

$$
\Omega^{i}=\mathrm{d} \omega^{i}-\omega^{j} \wedge \omega_{j}^{i},
$$

qui se réduit, en vertu de (1.24), à

$$
\Omega^{a}=\mathrm{d} \omega^{a}-\omega^{b} \wedge \omega_{b}^{a}, \quad \Omega^{u}=\mathrm{d} \omega^{u}-\omega^{v} \wedge \omega_{v}^{u} .
$$

Dans une intersection $U_{\alpha} \cap U_{\beta}$ les $\left(\Omega^{a}, \Omega^{u}\right)$ ont les mêmes formules de transformation que les $\left(\omega^{a}, \omega^{u}\right)[1]$ et, comme ces formules sont (1.3), il suit que le type des formes $\Omega^{a}$ et le type des formes $\Omega^{u}$ sont invariants.

La troisième condition annoncée ci-dessus est que les formes $\Omega^{a}$ soient du type $(0,2)$ et $\Omega^{u}$ du type $(2,0)$. Ceci donne

$$
\begin{aligned}
& \Gamma_{b c}^{a}-\Gamma_{c b}^{a}=\lambda_{b c}^{a}, \quad \Gamma_{b u}^{a}=\lambda_{b u}^{a}, \\
& \Gamma_{v w}^{u}-\Gamma_{w v}^{u}=\lambda_{v w}^{u}, \quad \Gamma_{v b}^{u}=\lambda_{v b}^{u} .
\end{aligned}
$$

De cette troisième condition il suit

$$
\Omega^{a}=0\left(\bmod . \omega^{u}\right), \quad \Omega^{u}=0\left(\bmod . \omega^{a}\right),
$$

donc, d'après (1.26)

$$
\mathrm{d} \omega^{a}=\omega^{b} \wedge \omega_{b}^{a}\left(\bmod . \omega^{u}\right), \quad \mathrm{d} \omega^{u}=\omega^{v} \wedge \omega_{v}^{u}\left(\bmod . \omega^{a}\right) .
$$

Des équations (1.25) et (1.29) on obtient évidemment

$$
\Gamma_{b c}^{a}=\gamma_{b c}^{a}, \quad \Gamma_{v w}^{u}=\gamma_{v w}^{u},
$$

relations qui sont compatibles avec $(1.27),(1.28)$ et qui achèvent la détermination de la connexion désirée.

En résumant, on a le 
Théorème 1.2. Sur la v.r.p.f. $V$, il y a une connexion unique vérifiant les conditions: a) elle induit dans les fibrés $D(V)$ et $D^{\perp}(V)$ des connexions qui sont euclidiennes le long des courbes tangentes à $\mathscr{D}$, ou, respectivement, $\mathscr{D}^{\perp}, \mathrm{b}$ ) les premières $n$ formes de torsion de cette connexion ont le type $(0,2)$ et les dernières $m$ ont le type $(2,0)$. Les coefficients de cette connexions sont

$$
\Gamma_{b c}^{a}=\gamma_{b c}^{a}, \quad \Gamma_{v w}^{u}=\gamma_{v w}^{u}, \quad \Gamma_{b u}^{a}=\lambda_{b u}^{a}, \quad \Gamma_{v b}^{u}=\lambda_{v b}^{u}, \quad \Gamma_{u i}^{a}=0, \quad \Gamma_{a i}^{u}=0 .
$$

La connexion donnée par le théorème 1.2 sera nommée la seconde connexion de la v.r.p.f.

Les formes de torsion de la seconde connexion de $V$ sont

$$
\Omega^{a}=-\frac{1}{2} \lambda_{u v}^{a} \omega^{u} \wedge \omega^{v}, \quad \Omega^{u}=-\frac{1}{2} \lambda_{a b}^{u} \omega^{a} \wedge \omega^{b},
$$

d'où il suit le

Théorème 1.3. La seconde connexion d'une v.r.p.f. est sans torsion si et seulement si $\mathscr{D}$ et $\mathscr{D}^{\perp}$ sont intégrables.

Considérons maintenant les formes

$$
\omega_{j}^{i}-\bar{\omega}_{j}^{i}=\eta_{j}^{i}=a_{j k}^{i} \omega^{k} .
$$

Compte tenu des expressions de $\omega$ et $\bar{\omega}$, il résulte, après des calculs

$$
\begin{aligned}
& \eta_{b}^{a}=-\gamma_{u b}^{a} \omega^{u}, \quad \eta_{v}^{u}=-\gamma_{a v}^{u} \omega^{a}, \\
& \eta_{u}^{a}=-\gamma_{u b}^{a} \omega^{b}+\gamma_{a v}^{u} \omega^{v}, \quad \eta_{a}^{u}=\gamma_{u b}^{a} \omega^{b}-\gamma_{a v}^{u} \omega^{v} .
\end{aligned}
$$

Donc, $\eta_{j}^{i}=0$ si et seulement si

$$
\gamma_{u b}^{a}=\gamma_{a v}^{u}=0
$$

et, en considérant encore les conditions (1.14), on trouve le

Théorème 1.4. La seconde connexion d'une v.r.p.f. coïncide avec la première si et seulement si cette dernière induit des connexions dans les fibrés transversal et structurel de la variété.

Du théorème 1.1, on sait qu'il suffit que la première connexion induise une connexion dans un de ces deux fibrés et que dans ce cas les distributions $\mathscr{D}$ et $\mathscr{D}^{\perp}$ sont intégrables.

La seconde connexion d'une v.r.p.f. a encore d'autres propriétés intéressantes. Ainsi, une courbe autoparallèle de celle-ci est tangente dans tous ses points à l'une des distributions $\mathscr{D}, \mathscr{D}^{\perp}$ si et seulement si elle l'est dans un de ses points. Les formes de courbure de la connexion sont

$$
\Omega_{b}^{a}=\mathrm{d} \omega_{b}^{a}-\omega_{b}^{c} \wedge \omega_{c}^{a}, \quad \Omega_{v}^{u}=\mathrm{d} \omega_{v}^{u}-\omega_{v}^{w} \wedge \omega_{w}^{u}, \quad \Omega_{u}^{a}=\Omega_{a}^{u}=0 .
$$


Ces formes donnent le tenseur de courbure qui peut être employé exactement comme dans la géométrie riemannienne pour définir une courbure sectionnelle de la seconde espèce. Comme dans le cas riemannien, celle-ci a la valeur constante $k$ si et seulement si $[1]$

$$
\Omega_{i}^{j}=k \omega^{i} \wedge \omega^{j},
$$

d'où, compte tenu de (1.35), on trouve $k=0$, etc.

Une remarque importante est que le type des formes reste invariant aux transformations des cobases de la forme (1.3), même si les matrices $p$ et $q$ ne sont pas orthogonales. Il suit que les coefficients de la seconde connexion peuvent être calculés analoguement dans les cobases, qui s'obtiennent par ces transformations plus générales.

\section{VARIÉTÉS RIEMANNIENNES FEUILLETÉES}

2.1. En gardant les notations du paragraphe précédent, nous dirons, par définition, que la v.r.p.f. $V$ est une variété riemannienne feuilletée (v.r.f.) si la distribution structurelle $\mathscr{D}$ est intégrable. Alors, les variétés intégrales maximales de $\mathscr{D}$ sont les feuilles de la v.r.f. Une v.r.f. est caractérisée analytiquement par la condition (1.11).

Déterminons maintenant les divers éléments considérés dans le $\S 1$, pour une v.r.f.

Tout d'abord, il est clair de la définition précédente (voir par exemple [8]), qu'il y a sur $V$ un atlas $\mathscr{A}=\left\{\left(U_{\alpha}, h_{\alpha}\right) / h_{\alpha}: U_{\alpha} \rightarrow R^{n+m}\right\}$, tel que pour chaque $U_{\alpha}$, si l'on note par $\left(x_{\alpha}^{a}, x_{\alpha}^{u}\right)$ les coordonnées locales correspondantes, le système de Pfaff $\omega^{a}$ soit équivalent au système $\mathrm{d} x^{a}$ et le système $\omega^{u}$ soit équivalent à un système de la forme $\theta^{u}=\mathrm{d} x^{u}+t_{a}^{u} \mathrm{~d} x^{a}$. Alors, la distribution $\mathscr{D}$ est définie par les équations

$$
\mathrm{d} x^{a}=0
$$

et la distribution complémentaire $\mathscr{D}^{\perp}$ par les équations

$$
\theta^{u}=\mathrm{d} x^{u}+t_{a}^{u} \mathrm{~d} x^{a}=0 .
$$

Evidemment, dans une intersection $U_{\alpha} \cap U_{\beta}$, le système $\mathrm{d} x_{\alpha}^{a}$ doit être équivalent à $\mathrm{d} x_{\beta}^{a}$, d'où on obtient pour les fonctions de transformation de l'atlas $\mathscr{A}$ les conditions fondamentales

$$
\frac{\partial x_{\beta}^{a}}{\partial x_{a}^{u}}=0
$$

qui expriment le fait que, dans $R^{n+m}$, les fonctions $x_{\beta}^{a}$ sont constantes sur les composantes connexes de l'intersection du domaine $h_{\alpha}\left(U_{\alpha} \cap U_{\beta}\right)$ avec les plans $x_{\alpha}^{a}=$ $=$ const.

Pour les formes $\theta^{u}$, on obtient les formules de transformation

$$
\theta_{\beta}^{u}=\frac{\partial x_{\beta}^{u}}{\partial x_{\alpha}^{v}} \theta_{\alpha}^{v}+\left(\frac{\partial x_{\beta}^{u}}{\partial x_{\alpha}^{b}}+t_{\beta}^{u} \frac{\partial x_{\beta}^{a}}{\partial x_{\alpha}^{b}}-t_{\alpha}^{v} \frac{\partial x_{\beta}^{u}}{\partial x_{\alpha}^{v}}\right) \mathrm{d} x_{\alpha}^{b},
$$


d'où, comme les systèmes $\theta_{\alpha}^{u}$ et $\theta_{\beta}^{u}$ doivent être équivalents, il résulte

$$
\begin{gathered}
\theta_{\beta}^{u}=\frac{\partial x_{\beta}^{u}}{\partial x_{\alpha}^{v}} \theta_{\alpha}^{v}, \\
t_{\beta}^{u}=\frac{\partial x_{\beta}^{u}}{\partial x_{\alpha}^{v}} \frac{\partial x_{\alpha}^{b}}{\partial x_{\beta}^{c}} t_{b}^{v}-\frac{\partial x_{\beta}^{u}}{\partial x_{\alpha}^{b}} \frac{\partial x_{\alpha}^{b}}{\partial x_{\beta}^{c}},
\end{gathered}
$$

la dernière formule étant la loi de transformation de $t_{a}^{u}$ (dans la déduction de celle-ci on emploie (2.3)).

On voit que les formes $\left(\mathrm{d} x^{a}, \theta^{u}\right)$ définissent des cobases de la variété $V$, qui sont adaptées à la structure presque-produit $\mathscr{P} \mathrm{du} \S 1$ (dans le sens que les distributions $\mathscr{D}$ et $\mathscr{D}^{\perp}$ sont définies par (2.1) et (2.2)) et; dans tout ce qui suit il sera plus convenable d'employer ces cobases au lieu de celles qui ont été employées dans le $\S 1$. Remarquons qu'alors les conditions d'intégrabilité de la distribution transversale $\mathscr{D}^{\perp}$ sont

$$
\frac{\partial}{\partial x^{b}} t_{a}^{u}-\frac{\partial}{\partial x^{a}} t_{b}^{u}-t_{b}^{v} \frac{\partial}{\partial x^{v}} t_{a}^{u}+t_{a}^{v} \frac{\partial}{\partial x^{v}} t_{b}^{u}=0 .
$$

Il est important de signaler que, en vue de la remarque finale du $\S 1$, le type des formes différentielles de $V$ est déterminé par leurs expressions dans ces nouvelles cobases.

On peut vérifier aisément, par un calcul direct, que les bases duales des cobases précédentes sont

$$
X_{a}=\frac{\partial}{\partial x^{a}}-t_{a}^{u} \frac{\partial}{\partial x^{u}}, \quad X_{u}=\frac{\partial}{\partial x^{u}},
$$

donc $\mathscr{D}$ est engendré par les $X_{u}$ et $\mathscr{D}^{\perp}$ par les $X_{a}$ et il suit encore que les fonctions de passage du fibré structurel sont $\left(\partial x_{\beta}^{u} / \partial x_{\alpha}^{v}\right)$ et celles du fibré transversal sont $\left(\partial x_{\beta}^{a} / \partial x_{\alpha}^{b}\right)$.

Les cobases considérées ci-dessus ne sont pas, en général, orthonormées. Si nous partons de l'expression de la métrique $g$ de $V$ à l'aide de l'atlas $\mathscr{A}$

$$
\mathrm{d} s^{2}=g_{i j} \mathrm{~d} x^{i} \mathrm{~d} x^{j},
$$

nous trouvons, avec (2.2)

$$
\mathrm{d} s^{2}=\left(g_{a b}-2 g_{a u} t_{b}^{u}+g_{u v} t_{a}^{u} t_{b}^{v}\right) \mathrm{d} x^{a} \mathrm{~d} x^{b}+2\left(g_{a u}-g_{u v} t_{a}^{v}\right) \mathrm{d} x^{a} \theta^{u}+g_{u v} \theta^{u} \theta^{v}
$$

et, en tenant compte de l'orthogonalité de $\mathscr{D}$ et $\mathscr{D}^{\perp}$, il résulte

$$
g_{a u}-g_{u v} t_{a}^{v}=0
$$

On voit alors que la métrique induite dans le fibré structurel est $g_{u v} \theta^{u} \theta^{v}$, donc $g_{u v}$ est une matrice non-dégénérée et nous pouvons introduire la matrice $\tilde{g}^{u v}$ - inverse 
de $g_{u v}$ - de telle manière qu'on ait

$$
t_{a}^{u}=\tilde{g}^{u v} g_{a v} .
$$

Finalement, nous obtenons l'expression de la métrique $g$ dans les cobases $\left(\mathrm{d} x^{a}, \theta^{u}\right)$

$$
\mathrm{d} s^{2}=h_{a b} \mathrm{~d} x^{a} \mathrm{~d} x^{b}+h_{u v} \theta^{u} \theta^{v},
$$

où

$$
h_{a b}=g_{a b}-\tilde{g}^{u v} g_{a u} g_{b v}, \quad h_{u v}=g_{u v} .
$$

Donc, la matrice du tenseur métrique, rapporté à nos cobases, est

$$
\left(\begin{array}{ll}
h_{a b} & 0 \\
0 & h_{u v}
\end{array}\right)
$$

et sa matrice inverse a la forme

$$
\left(\begin{array}{ll}
\tilde{h}^{a b} & 0 \\
0 & \tilde{h}^{u v}
\end{array}\right) .
$$

Il est encore important de remarquer que les deux termes du second membre de (2.10) donnent respectivement la métrique induite dans le fibré transversal et dans le fibré structurel.

2.2. La première connexion de la v.r.f. $V$ étant la connexion de Levi-Civita de la métrique $g$, on peut la calculer, de la manière usuelle, à l'aide des coefficients $g_{i j}$ de (2.8), dans les cobases $\left(\mathrm{d} x^{a}, \mathrm{~d} x^{u}\right)$ et puis, avec (2.2), on obtiendre cette connexion dans les cokses $\left(\mathrm{d} x^{a}, \theta^{u}\right)$.

Quant à la seconde connexion de $V$, pour la trouver dans les cobases $\left(\mathrm{d} x^{a}, \theta^{u}\right)$, nous noterons les formes de connexion par $\omega_{i}^{j}$ et

$$
\omega_{i}^{j}=\Gamma_{i a}^{j} \mathrm{~d} x^{a}+\Gamma_{i u}^{j} \theta^{u}
$$

et imposerons les trois conditions du $\S 1.3$.

La condition que cette connexion induise des connexions dans $D(V)$ et $D^{\perp}(V)$ est, comme au $\S 1.3$ :

$$
\omega_{u}^{a}=\omega_{a}^{u}=0 .
$$

Les conditions que ces connexions induites soeint euclidiennes (au sens du théorème 1.2) sont, somme dans le cas d'un espace de Riemann $[1,2]$,

$$
\begin{aligned}
& \mathrm{d} h_{a b}-\omega_{a}^{c} h_{c b}-\omega_{b}^{c} h_{a c}=0\left(\bmod \theta^{u}\right), \\
& \mathrm{d} h_{u v}-\omega_{u}^{w} h_{w v}-\omega_{v}^{w} h_{u w}=0\left(\bmod \mathrm{d} x^{a}\right) .
\end{aligned}
$$


Les formes de torsion de la connexion sont, compte tenu de (2.15),

$$
\begin{gathered}
\Omega^{a}=-\frac{1}{2}\left(\Gamma_{b c}^{a}-\Gamma_{c b}^{a}\right) \mathrm{d} x^{b} \wedge \mathrm{d} x^{c}-\Gamma_{b u}^{a} \mathrm{~d} x^{b} \wedge \theta^{u}, \\
\Omega^{u}=X_{b} t_{a}^{u} \mathrm{~d} x^{b} \wedge \mathrm{d} x^{a}+\left(\Gamma_{v a}^{u}-X_{v} t_{a}^{u}\right) \mathrm{d} x^{a} \wedge \theta^{v}- \\
-\frac{1}{2}\left(\Gamma_{v w}^{u}-\Gamma_{w v}^{u}\right) \theta^{v} \wedge \theta^{w},
\end{gathered}
$$

donc $\Omega^{a}$ a le type $(0,2)$ (et il est nul alors) si et seulement si

$$
\Gamma_{b c}^{a}=\Gamma_{c b}^{a}, \quad \Gamma_{b u}^{a}=0
$$

et $\Omega^{u}$ a le type $(2,0)$ si et seulement si

$$
\Gamma_{v w}^{u}=\Gamma_{w v}^{u}, \quad \Gamma_{v a}^{u}=\frac{\partial}{\partial x^{v}} t_{a}^{u} .
$$

Donc, pour achever la détermination de la seconde connexion de $V$, it faut encore calculer $\Gamma_{b c}^{a}$ et $\Gamma_{v w}^{u}$. Ler symétrie dans les indices inférieurs et les conditions (2.16) montrent que le calcul de ces coefficients est le même que le calcul des symboles de Christoffel. Il suit ainsi

$$
\begin{aligned}
& \Gamma_{b c}^{a}=\frac{1}{2} \tilde{h}^{a d}\left(X_{c} h_{b d}+X_{b} h_{c d}-X_{d} h_{b c}\right), \\
& \Gamma_{v w}^{u}=\frac{1}{2} \tilde{h}^{u t}\left(X_{w} h_{v t}+X_{v} h_{w t}-X_{t} h_{v w}\right) .
\end{aligned}
$$

En résumant, nous avons trouvé que les formes de la seconde connexion d'une v.r.f. sont

$$
\omega_{b}^{a}=\Gamma_{b c}^{a} \mathrm{~d} x^{c}, \quad \omega_{v}^{u}=\Gamma_{v w}^{u} \theta^{w}+\frac{\partial}{\partial x^{v}} t_{a}^{u} \mathrm{~d} x^{a}, \quad \omega_{u}^{a}=\omega_{a}^{u}=0,
$$

où $\Gamma_{b c}^{a}$ et $\Gamma_{v w}^{u}$ sont donnés par (2.20).

Les formes de la même connexion dans les cobases naturelles $\left(\mathrm{d} x^{a}, \mathrm{~d} x^{u}\right)$ de l'atlas $\mathscr{A}$ sont données par (2.21) et (2.2), en employant la loi de transformation des formes de connexion

$$
\tilde{\omega}_{a}^{b}=\omega_{a}^{b}, \quad \tilde{\omega}_{u}^{v}=\omega_{u}^{v}, \quad \tilde{\omega}_{u}^{a}=0, \quad \tilde{\omega}_{a}^{u}=\mathrm{d} t_{a}^{u}+t_{a}^{v} \omega_{v}^{u}-t_{b}^{u} \omega_{a}^{b} .
$$

Si l'on pose

il résulte

$$
\tilde{\omega}_{i}^{j}=\Pi_{i k}^{j} \mathrm{~d} x^{k}
$$

$$
\begin{gathered}
\Pi_{a c}^{b}=\Gamma_{a c}^{b}, \quad \Pi_{a u}^{b}=0, \quad \Pi_{u a}^{v}=\frac{\partial}{\partial x^{u}} t_{a}^{v}+t_{a}^{w} \Gamma_{u w}^{v}=\Pi_{a u}^{v}, \\
\Pi_{u w}^{v}=\Gamma_{v w}^{u}, \quad \Pi_{u b}^{a}=\Pi_{u v}^{a}=0, \\
\Pi_{a b}^{u}=\frac{\partial}{\partial x^{b}} t_{a}^{u}+t_{a}^{v} \frac{\partial}{\partial x^{v}} t_{b}^{u}+\Gamma_{v w}^{u} t_{a}^{v} t_{b}^{w}-\Gamma_{a b}^{c} t_{c}^{u} .
\end{gathered}
$$


Les formes de torsion de la connexion envisagée sont, selon les formules (2.17), (2.18) et (2.19)

$$
\Omega^{a}=0, \quad \Omega^{u}=X_{a} t_{b}^{u} \mathrm{~d} x^{a} \wedge \mathrm{d} x^{b},
$$

d'où les composantes du tenseur de torsion dans les cobases $\left(\mathrm{d} x^{a}, \theta^{u}\right)$

$$
T_{i j}^{a}=0, \quad T_{a b}^{u}=-\frac{1}{2}\left(X_{a} t_{b}^{u}-X_{b} t_{a}^{u}\right), \quad T_{a v}^{u}=0, \quad T_{v w}^{u}=0 .
$$

Les formes de courbure de la seconde connexion seront calculées par les formules usuelles (dans la notation de [3])

$$
\Omega_{i}^{j}=\mathrm{d} \omega_{i}^{j}-\omega_{i}^{k} \wedge \omega_{k}^{j}=-\frac{1}{2} R_{i k h}^{j} \omega^{k} \wedge \omega^{h} .
$$

En employant les formules (2.21), on trouve les composantes du tenseur de courbure dans les cobases $\left(\mathrm{d} x^{a}, \theta^{u}\right)$ et les bases $\left(X_{a}, X_{u}\right)$

$$
\begin{aligned}
& R_{b u v}^{a}=0, \quad R_{b c u}^{a}=X_{u} \Gamma_{b c}^{a}, \quad R_{u i j}^{a}=0, \quad R_{a i j}^{u}=0, \\
& R_{b c d}^{a}=X_{d} \Gamma_{b c}^{a}-X_{c} \Gamma_{b d}^{a}+\Gamma_{b d}^{e} \Gamma_{e d}^{a}-\Gamma_{b d}^{e} \Gamma_{e c}^{a}, \\
& R_{u w s}^{v}=X_{s} \Gamma_{u w}^{v}-X_{w} \Gamma_{u s}^{v}+\Gamma_{u w}^{t} \Gamma_{t s}^{v}-\Gamma_{u s}^{t} \Gamma_{t w}^{v}, \\
& R_{u c s}^{v}=X_{s} X_{u} t_{c}^{v}+\Gamma_{u w}^{v} X_{s} t_{c}^{w}-X_{c} \Gamma_{u s}^{v}+\Gamma_{w s}^{v} X_{u} t_{c}^{w}-\Gamma_{u s}^{w} X_{w} t_{c}^{v}, \\
& R_{u a b}^{v}=\Gamma_{u w}^{v}\left(X_{b} t_{a}^{w}-X_{a} t_{b}^{w}\right)+X_{b} X_{u} t_{a}^{v}-X_{a} X_{u} t_{b}^{v}+X_{u} t_{a}^{w} \cdot X_{w} t_{b}^{v}-X_{u} t_{b}^{w} \cdot X_{w} t_{a}^{v} .
\end{aligned}
$$

D'ici on peut déduire aisément les composantes du tenseur de Ricci, mais nous nous contentons de faire la remarque importante, qu'on peut démontrer exactement comme pour un espace de Riemann, que les composantes $R_{u v}$ sont symétriques dans les indices $u$ et $v$. Mais, en général, les $R_{i j}$ ne sont pas symétriques. Par exemple, on peut voir que les $R_{a b}$ sont symétriques dans le cas où $\mathscr{D}^{\perp}$ est intégrable et dans le cas où $\operatorname{det}\left(h_{a b}\right)$ ne dépend pas des variables $x^{u}$ ce qui aura lieu en particulier pour les espaces de Reinhart qui seront considérés plus tard.

Des considérations de ce paragraphe, il s'ensuit le fait remarquable que les coefficients $\Gamma_{v w}^{u}$ (et partiellement les $\Gamma_{b c}^{a}$ ) ont un comportement analogue à celui des symboles de Christoffel d'un espace de Riemann. Ceci s'explique par le fait que pour $x^{a}=$ $=$ const. $h_{u v} \theta^{u} \theta^{v}$ est la métrique riemannienne locale, d'une feuille. Ce comportement des coefficients $\Gamma$ peut être remarqué aussi dans d'autres questions. Par exemple, on voit qu' à une transformation conforme de la métrique (2.8) les fonctions $t_{a}^{u}$ restent invariantes selon (2.9), donc les formes $\theta^{u}$ restent invariantes et, parmi les coefficients de la seconde connexion, les seuls qui changent sont $\Gamma_{b c}^{a}$ et $\Gamma_{v w}^{u}$, leurs formules de transformation étant les mêmes que dans le cas d'une transformation conforme d'un espace de Riemann.

2.3. Soit de nouveau la v.r.f. $V$, douée de l'atlas $\mathscr{A}$ du $\S 2.1$. Les formules fondamentales (2.3) vérifiées par cet atlas nous conduisent à définir de nouveaux éléments importants sur $V$. 
Ainsi, une fonction différentiable $\varphi$ définie sur un ouvert de $V$ sera appelée feuilletée si

$$
\frac{\partial \varphi}{\partial x^{u}}=0
$$

ces conditions étant invariantes à cause de (2.3). Nous pouvons alors considérer le faisceau $\Phi$ des germes des fonctions feuilletées sur $V$ qui est un sous-faisceau du faisceau $\mathscr{F}$ des germes des fonctions différentiables sur $V$. Remarquons que les formules (2.27) montrent que chaque point du domaine de définition de $\varphi$ a un voisinage dans lequel $\varphi$ a l'expression $\varphi\left(x^{a}\right)$, donc les germes de $\Phi$ peuvent se représenter dans cette manière et les fonctions feuilletées sont des fonctions constantes sur les feuilles de $V$. Le faisceau $\Phi$ n'est pas, en général, fin et c'est pourquoi il joue un rôle important pour la variété $V$.

Les considérations de ci-dessus ne dépendent pas de la métrique de $V$; elles sont donc valables pour le cas où $V$ est simplement une variété feuilletée, c'est-à-dire une variété qui possède un atlas $\mathscr{A}$ vérifiant les conditions (2.3). De plus, elles admettent une généralisation immédiate.

En effet, soit $\bar{V}^{p+q}$ une autre variété feuilletée, de codimension $p$, et soient $\left(y^{x}, y^{\sigma}\right)$ $(\alpha=1, \ldots, p ; \sigma=p+1, \ldots, p+q)$ les coordonnées locales de l'atlas $\mathscr{A}$ de $\bar{V}$. Une application différentiable $\varphi: V \rightarrow \bar{V}$ sera dite feuilletée si l'expression locale de cette application vérifie les conditions

$$
\frac{\partial y^{\alpha}}{\partial x^{u}}=0
$$

conditions qui sont invariantes aux changements des cartes locales des atlas $\mathscr{A}$ de $V$ et de $\bar{V}$, à cause de (2.3).

Ainsi, par exemple, si nous considérons le fibré tangent de la v.r.f. $V, T(V)$, alors à l'atlas $\mathscr{A}$ de $V$ correspond sur $T(V)$ un atlas tel qu'aux coordonnées locales $\left(x^{a}, x^{u}\right)$ de $V$ correspondent les coordonnées locales $\left(x^{a}, x^{u}, \xi^{a}, \xi^{u}\right)$, où $\xi^{a}$ et $\xi^{u}$ sont les coordonnées des vecteurs tangents par rapport aux bases $\left(X_{a}, X_{u}\right)$. Les changements de coordonnées de cet atlas seront (comme suite de (2.3) et (2.4))

$$
x_{\beta}^{a}=x_{\beta}^{a}\left(x_{\alpha}^{b}, x_{\alpha}^{v}\right), \quad x_{\beta}^{u}=x_{\beta}^{u}\left(x_{\alpha}^{b}, x_{\alpha}^{v}\right), \quad \xi_{\beta}^{a}=\frac{\partial x_{\beta}^{a}}{\partial x_{\alpha}^{b}} \xi_{\alpha}^{b}, \quad \xi_{\beta}^{u}=\frac{\partial x_{\beta}^{u}}{\partial x_{\alpha}^{v}} \xi_{\alpha}^{v},
$$

d'où il suit qu'en considérant les $\left(x^{a}, \xi^{a}\right)$ comme premières $2 n$ coordonnées et les $\left(x^{u}, \xi^{u}\right)$ comme dernières $2 m$ coordonnées, on a sur $T(V)$ une structure feuilletée, dont la distribution structurelle est définie par

$$
\mathrm{d} x^{a}=0, \quad \mathrm{~d} \xi^{a}=0 .
$$

De plus, si l'on considère sur $T(V)$, par exemple, la métrique riemannienne de Sasaki [10], on voit que $T(V)$ est une v.r.f. de codimension $2 n$. 
Alors il est naturel d'appeler champ de vecteurs feuilleté sur $V$ un champ de vecteurs tangents à $V$ qui est défini comme une application feuilletée de $V$ à $T(V)$, c'est-à-dire une section feuilletée de $T(V)$. Bien entendu, on peut considérer le faisceau $\Xi$ des germes des champs de vecteurs feuilletés. On sait qu'un champ de vecteurs défini par les coordonnées locales $\left(\xi^{a}, \xi^{u}\right)$ est feuilleté si et seulemenet si

$$
\frac{\partial \xi^{a}}{\partial x^{u}}=0
$$

Analoguement, il y a une structure feuilletée sur le fibré transversal $D^{\perp}(V)$, définie par l'atlas ayant pour premières coordonnées locales les variables $\left(x^{a}, \xi^{a}\right)$ et pour dernières les variables $x^{u}$ ( $\xi^{a}$ sont les coordonnées dans les fibres de $D^{\perp}(V)$; par rapport aux bases $X_{a}$ ), ce qui transforme $D^{\perp}(V)$ dans une v.r.f. de codimension $2 n$ (avec une métrique quelconque). Donc, on peut parler de sections feuilletées dans le fibré $D^{\perp}(V)$, sections qui sont caractérisées aussi par (2.30) et qui définissent un faisceau $\Lambda$ de germes.

Pour faire une remarque intéressante, notons encore par $M$ le faisceau des germes des sections différentiables du fibré structụrel $D(V)$. Alors, on obtient aisément une suite exacte de faisceaux sur $V$

$$
0 \rightarrow M \stackrel{i}{\rightarrow} \Xi \stackrel{p}{\rightarrow} \Lambda \rightarrow 0,
$$

où les homomorphismes $i$ et $p$ sont définis par

$$
i\left(\xi^{u}\right)=\left(0, \xi^{u}\right), \quad p\left(\xi^{a}, \xi^{u}\right)=\left(\xi^{a}\right)
$$

et, de la suite exacte de cohomologie correspondante, compte tenu du fait que $M$ est un faisceau fin sur la variété paracompacte $V$, on obtient pour les groupes de cohomologie la relation

$$
H^{q}(V, \Xi)=H^{q}(V, \Lambda), \quad(q \geqq 1) .
$$

La définition des champs de vecteurs feuilletés peut être transposée à d'autres objets géométriques aussi. Ainsi, pour les formes différentielles du type $(p, 0)$, on voit que la condition que les composantes locales de celles-ci soient des fonctions feuilletées est invariante et nous dirons que les formes qui la satisfont sont des formes différentielles feuilletées de degré $p$. Ce sont justement les formes de type basique, dont la cohomologie a été étudiée par Reinahrt [9].

Puis, une condition analogue peut être imposée à la métrique de la v.r.f. En effet, il est simple de voir que les conditions

$$
\frac{\partial h_{a b}}{\partial x^{u}}=0
$$

sont invariantes à des changements de coordonnées par rapport aux cobases $\left(\mathrm{d} x^{a}\right.$, 
$\theta^{u}$ ). Les v.r.f., dont la métrique satisfait à (2.34) (on pourrait dire qu'elle est feuilletée), forment une classe très importante de v.r.f. Cette classe a été étudiée par Reinhart [8] et c'est pourquoi nous appelons une pareille v.r.f. espace de Reinhart.

Remarquons que, pour un espace de Reinhart, on peut remplacer dans la première des formules (2.20) les $X_{c}$ par $\partial / \partial x^{c}$ et qu'on a aussi

$$
\frac{\partial}{\partial x^{u}} \Gamma_{b c}^{a}=0
$$

d'où, avec (2.26),

$$
R_{b c u}^{a}=0 .
$$

Il est à remarquer aussi que, dans ce cas, la première des relations (2.17) est vérifiée pour n'importe quelle courbe de la variété $V$.

\section{FORMES DIFFÉRENTIELLES ET COHOMOLOGIE À COEFFICIENTS DANS LE FAISCEAU $\Phi$ D'UNE V.R.F.}

3.1. Dans tout ce qui suit, nous gardons les notations du $\S 2$. Pour tous les tenseurs, les composantes seront considérées par rapport aux bases $\left(X_{a}, X_{u}\right)$ et aux cobases $\left(\mathrm{d} x^{a}, \theta^{u}\right)$.

Dans le $\S 2.3$, nous avons défini le faisceau $\Phi$ de germes de fonctions feuilletées sur la variété feuilletée $V$, qui n'est pas, en général, fin et qui donne donc lieu à une théorie de cohomologie non triviale sur $V$. Il est clair que celle-ci est importante dans l'étude de la variété $V$ et nous voulons calculer les groupes $H^{q}(V, \Phi)$. D'une façon plus générale, notons par $\Phi^{p}$ le faisceau des germes des formes différentielles feuilletées de degré $p$ et proposons-nous de calculer les groupes de cohomologie $H^{q}\left(V, \Phi^{p}\right)$. A ce but, nous trouverons une résolution fine du faisceau $\Phi^{p}$, à l'aide des formes différentielles de la variété $V$; dans le cas $p=0$, notre résolution est isomorphe à celle donnée pour le faisceau $\Phi$ par SPENCER [11].

Considérons une forme différentielle du type $(p, q)$

$$
\varphi=\frac{1}{p ! q !} \varphi_{a_{1} \ldots a_{p} u_{1} \ldots u_{q}} \mathrm{~d} x^{a_{1}} \wedge \ldots \wedge \mathrm{d} x^{a_{p}} \wedge \theta^{u_{1}} \wedge \ldots \wedge \theta^{u_{q}}
$$

et calculons sa différentielle extérieure $\mathrm{d} \varphi$. On trouve alors

$$
\mathrm{d} \varphi=\partial \varphi+\mathrm{d}^{\prime} \varphi+\mathrm{d}^{\prime \prime} \varphi
$$

où

$$
\begin{aligned}
\partial \varphi= & \frac{(-1)^{p}}{(q-1) ! p !(p+2) !} \varepsilon_{b_{1} \ldots a_{p+2}}^{b c a_{1} \ldots a_{p}} \varphi_{a_{1} \ldots a_{p} u v_{1} \ldots v_{q-1}} X_{b} t_{c}^{u} \mathrm{~d} x^{b_{1}} \wedge \ldots \wedge \mathrm{d} x^{b_{p+2}} \wedge \\
& \wedge \theta^{v_{1}} \wedge \ldots \wedge \theta^{v_{q-1}},
\end{aligned}
$$




$$
\begin{aligned}
\mathrm{d}^{\prime} \varphi= & \frac{1}{p ! q !(p+1) !} \varepsilon_{b_{1} \ldots b_{p+1}}^{b a_{1} \ldots a_{p}}\left(X_{b} \varphi_{a_{1} \ldots a_{p} u_{1} \ldots u_{q}}-\right. \\
& \left.-\frac{1}{(q-1) !} \varepsilon_{u_{1} \ldots u_{q}}^{v v_{1} \ldots v_{q-1}} \varphi_{a_{1} \ldots a_{p} w v_{1} \ldots v_{q}-1} \frac{\partial}{\partial x^{v}} t_{b}^{w}\right) \mathrm{d} x^{b_{1}} \wedge \ldots \wedge \mathrm{d} x^{b_{p}+1} \wedge \\
& \wedge \theta^{u_{1}} \wedge \ldots \wedge \theta^{u_{q}}
\end{aligned}
$$

$$
\begin{aligned}
\mathrm{d}^{\prime \prime} \varphi= & \frac{(-1)^{p}}{p ! q !(q+1) !} \varepsilon_{u_{1} \ldots u_{q}+1}^{v v_{1} \ldots v_{q}} \frac{\partial \varphi_{a_{1} \ldots a_{p} v_{1} \ldots v_{q}}}{\partial x^{v}} \mathrm{~d} x^{a_{1}} \wedge \ldots \wedge \mathrm{d} x^{a_{p}} \wedge \\
& \wedge \theta^{u_{1}} \wedge \ldots \wedge \theta^{u_{q+1}} .
\end{aligned}
$$

Dans ces formules, les $\varepsilon_{\ldots .}^{\ldots}$ sont les symboles de Kronecker et on peut trouver les règles de calcul avec ceux-ci, par exemple, dans [5].

On voit que la forme $\partial \varphi$ a le type $(p+2, q-1), \mathrm{d}^{\prime} \varphi$ a le type $(p+1, q)$ et $\mathrm{d}^{\prime \prime} \varphi$ a le type $(p, q+1)$, donc les opérateurs $\partial, \mathrm{d}^{\prime}, \mathrm{d}^{\prime \prime}$ ont respectivement le type $(2,-1)$, $(1,0)$ et $(0,1)$, c'est-à-dire, si l'on note par $A^{p q}$ l'espace des formes du type $(p, q)$ sur $V$, ces opérateurs sont des homomorphismes

$$
\partial: A^{p q} \rightarrow A^{p+2, q-1}, \quad \mathrm{~d}^{\prime}: A^{p q} \rightarrow A^{p+1, q}, \quad \mathrm{~d}^{\prime \prime}: A^{p q} \rightarrow A^{p, q+1} .
$$

En considérant la relation

$$
\mathrm{d}^{2}=\left(\partial+\mathrm{d}^{\prime}+\mathrm{d}^{\prime \prime}\right)^{2}=0
$$

et en annulant les termes des différents types qui y interviennent, on obtient

$$
\begin{gathered}
\mathrm{d}^{\prime \prime 2}=0, \quad \partial^{2}=0, \quad \mathrm{~d}^{\prime 2}+\mathrm{d}^{\prime \prime} \partial+\partial \mathrm{d}^{\prime \prime}=0, \quad \mathrm{~d}^{\prime} \mathrm{d}^{\prime \prime}+\mathrm{d}^{\prime \prime} \mathrm{d}^{\prime}=0, \\
\mathrm{~d}^{\prime} \partial+\partial \mathrm{d}^{\prime}=0 .
\end{gathered}
$$

Par la même méthode, en considérant $\mathrm{d}(\varphi \wedge \psi)$, on trouve

$$
\mathrm{d}^{\prime \prime}(\varphi \wedge \psi)=\mathrm{d}^{\prime \prime} \varphi \wedge \psi+(-1)^{\operatorname{deg} \varphi} \varphi \wedge \mathrm{d}^{\prime \prime} \psi
$$

et des règles analogues pour $\partial$ et $\mathrm{d}^{\prime}$.

Il est important de remarquer, comme suite de la formule (3.5) qu'une forme $\varphi$ est feuilletée si et seulement si elle a le type $(p, 0)(p=0, \ldots, n)$ et vérifie la condition

$$
\mathrm{d}^{\prime \prime} \varphi=0 \text {. }
$$

Une autre propriété importante de l'opérateur d" est qu'il vérifie un lemme de Poincaré, c'est-à-dire qu'on a le

Théorème 3.1. Soit $\varphi$ une forme du type $(p, q)(q \geqq 1)$ définie sur un voisinage de la variété $V$ et soit $\mathrm{d}^{\prime \prime} \varphi=0$. Alors, il y a sur le voisinage précédent, une forme $\alpha d u$ type $(p, q-1)$ et telle que $\varphi=\mathrm{d}^{\prime \prime} \alpha$. 
La démonstration sera faite par induction après l'indice $k$ qui est défini par la condition que la forme $\varphi$, qui est représentée par (3.1), ne contienne pas $\mathrm{d} x^{k+1}, \ldots, \mathrm{d} x^{n}$.

Pour $k=0$, on a

$$
\varphi=\frac{1}{q !} \varphi_{u_{1} \ldots u_{q}} 0^{u_{1}} \wedge \ldots \wedge \theta^{u_{q}}
$$

d'où il suit

$$
\mathrm{d}^{\prime \prime} \varphi=\mathrm{d} \varphi\left(\operatorname{modulo} \mathrm{d} x^{1}, \ldots, \mathrm{d} x^{n}\right)
$$

et, si $\mathrm{d}^{\prime \prime} \varphi=0$, nous avons avec le lemme de Poincaré dans l'espace des variables $x^{u}, x^{a}$ étant considérés comme des paramètres

$$
\varphi=\mathrm{d} \alpha\left(\operatorname{modul} l \mathrm{~d} x^{1}, \ldots, \mathrm{d} x^{n}\right),
$$

où $\alpha$ est une certaine forme du type $(0, q-1)$.

Il résulte alors que $\varphi$ est de la forme

$$
\varphi=\mathrm{d} \alpha+\sum_{a} \beta^{a} \wedge \mathrm{d} x^{a}
$$

et si l'on tient compte que $\varphi$ est du type $(0, q)$ cette expression se réduit à

$$
\varphi=\mathrm{d}^{\prime \prime} \alpha \text {, }
$$

ce qu'affirme notre théorème.

Supposons maintenant que l'affirmation du théorème est vraie pour les indices $k<h$ et soit $\varphi$ une forme du type $(p, q)$, qui ne contient pas $\mathrm{d} x^{h+1}, \ldots, \mathrm{d} x^{n}$. Alors on a

$$
\varphi=\mathrm{d} x^{h} \wedge \lambda+\mu
$$

où $\lambda$ et $\mu$ sont des formes qui ne contiennent pas $\mathrm{d} x^{h}, \mathrm{~d} x^{h+1}, \ldots, \mathrm{d} x^{n}$. Il suit

$$
\mathrm{d}^{\prime \prime} \varphi=-\mathrm{d} x^{h} \wedge \mathrm{d}^{\prime \prime} \lambda+\mathrm{d}^{\prime \prime} \mu,
$$

donc $\mathrm{d}^{\prime \prime} \varphi=0$ implique

$$
\mathrm{d}^{\prime \prime} \mu=0, \quad \mathrm{~d}^{\prime \prime} \lambda=0 .
$$

Selon l'hypothèse inductive, on trouve alors qu'il y a (sur le voisinage envisagé) une forme $\sigma$ du type $(p, q-1)$ et une forme $\tau$ du type $(p-1, q-1)$, telles que

$$
\mu=\mathrm{d}^{\prime \prime} \sigma, \quad \lambda=\mathrm{d}^{\prime \prime} \tau .
$$

Ceci donne enfin

$$
\varphi=\mathrm{d}^{\prime \prime}\left(-\mathrm{d} x^{k+1} \wedge \tau+\sigma\right)
$$

ce qui achève la démonstration du théorème 3.1.

Maintenant, la solution du problème que nous avons posé au début de ce para- 
graphe est immédiate. En effet, notons par $\mathscr{A}^{p q}$ le faisceau des germes des formes différentielles du type $(p, q)$ sur $V$ et considérons la suite de faisceaux

$$
0 \rightarrow \Phi^{p} \stackrel{i}{\rightarrow} \mathscr{A}^{p, 0} \stackrel{\mathrm{d}^{\prime \prime}}{\rightarrow} \mathscr{A}^{p, 1} \stackrel{\mathrm{d}^{\prime \prime}}{\rightarrow} \ldots \stackrel{\mathrm{d}^{\prime \prime}}{\rightarrow} \mathscr{A}^{p, m} \rightarrow 0,
$$

où $i$ est l'injection canonique et $\mathrm{d}^{\prime \prime}$ sont les homomorphismes définis par l'opérateur $\mathrm{d}^{\prime \prime}$ de ci-dessus. Cette suite est exacte à cause de la caractérisation (3.8) des formes feuilletées, de la propriété $\mathrm{d}^{\prime \prime 2}=0$ et du théorème 3.1. Ensuite, les faisceaux $\mathscr{A}^{p q}$ sont fins, donc (3.9) est une résolution fine du faisceau $\Phi^{p}$ et si nous notons par $Z^{p q}$ l'espace des formes différentielles, définies globalement sur $V$ et $\mathrm{d}^{\prime \prime}$-fermées il suit le

Théorème 3.2. $H^{q}\left(V, \Phi^{p}\right)=Z^{p q} / \mathrm{d}^{\prime \prime} A^{p, q-1}$.

C'est justement le résultat désiré. En particulier, nous avons

$$
H^{q}(V, \Phi)=Z^{0 q} / \mathrm{d}^{\prime \prime} A^{0, q-1} .
$$

Mentionnons encore le suivant

Corollaire. $H^{q}\left(V, \Phi^{p}\right)=0$, pour $q>m$.

3.2. Nous continuous l'étude de la cohomologie de $V$ à valeurs dans $\Phi^{p}$ par les méthodes de la théorie des formes harmoniques (voir par exemple [3] et [5]). A ce but, nous supposons dorénavant que $V$ est une v.r.f. compacte et orientée, l'orientation étant telle que la forme $\mathrm{d} x^{1} \wedge \ldots \wedge \mathrm{d} x^{n} \wedge \theta^{n+1} \wedge \ldots \wedge \theta^{n+m}$ soit positive. Nous supposons connue la théorie classique des formes harmoniques, telle comme elle est exposée, par exemple, dans [5] et [3] et nous employons les notations de ces deux livres.

Notons

$$
h_{1}=\operatorname{det}\left(h_{a b}\right), \quad h_{2}=\operatorname{det}\left(h_{u v}\right)
$$

Alors, l'élément de volume de $V$ est

$$
\mathrm{d} V=\sqrt{ }\left(h_{1} h_{2}\right) \mathrm{d} x^{1} \wedge \ldots \wedge \mathrm{d} x^{n} \wedge \theta^{n+1} \wedge \ldots \wedge \theta^{n+m}
$$

et les composantes de cette forme extérieure du type $(n, m)$ seront notées par $\eta_{\ldots}$.

Maintenant, en considérant la forme $\varphi$ donnée par (3.1) et en appliquant l'opérateur $*$, à l'aide de la formule qu'on trouve dans [5], il résulte

$$
\begin{aligned}
* \varphi= & \frac{(-1)^{(n-p) q}}{(n-p) !(m-q) ! p ! q !} \tilde{h}^{a_{1} b_{1}} \ldots \tilde{h}^{a_{p} b_{p}} \tilde{h}^{u_{1} v_{1}} \ldots \tilde{h}^{u_{q} v_{q}} . \\
& \cdot \eta_{b_{1} \ldots b_{p} c_{1} \ldots c_{n-p} v_{1} \ldots v_{q} w_{1} \ldots w_{m-q}} \varphi_{a_{1} \ldots a_{p} u_{1} \ldots u_{q}} \\
& . \mathrm{d} x^{c_{1}} \wedge \ldots \wedge \mathrm{d} x^{c_{n-p}} \wedge \theta^{w_{1}} \wedge \ldots \wedge \theta^{w_{m-q}} .
\end{aligned}
$$


On a aussi

$$
*^{-1}=(-1)^{(p+q)(n+m-p-q)} * .
$$

De (3.13), on voit que l'opérateur * transforme une forme du type $(p, q)$ dans une forme du type $(n-p, m-q)$.

On sait que, dans la théorie des formes harmoniques, on introduit le produit scalaire de deux formes quelconques $\varphi$ et $\psi$ par

$$
(\varphi, \psi)=\int_{V} \varphi \wedge * \psi .
$$

Si $\varphi$ et $\psi$ sont deux formes du type $(p, q)$, il résulte de (3.13)

$$
(\varphi, \psi)=\frac{1}{p ! q !} \int_{V} \varphi_{a_{1} \ldots a_{p} u_{1} \ldots u_{q}} \psi^{a_{1} \ldots a_{p} u_{1} \ldots u_{q}} \mathrm{~d} V,
$$

où $\mathrm{d} V$ est celui de (3.12) et on a employé l'élévement usuel des indices pour un espace de Riemann.

Considérons maintenant l'opérateur $\delta$, qui est donné par

$$
\delta=(-1)^{p+q} *^{-1} \mathrm{~d} *=\tilde{\partial}+\delta^{\prime}+\delta^{\prime \prime},
$$

où

$$
\begin{gathered}
\tilde{\partial}=(-1)^{p+q} *^{-1} \partial *, \quad \delta^{\prime}=(-1)^{p+q} *^{-1} \mathrm{~d}^{\prime} *, \\
\delta^{\prime \prime}=(-1)^{p+q} *^{-1} \mathrm{~d}^{\prime \prime} *
\end{gathered}
$$

sont des opérateurs ayant respectivement le type $(-2,1),(-1,0),(0,-1)$.

Un calcul tout à fait analogue à celui de la théorie classique (voir [5]), qui tient compte de la formule de Stokes, donne

$$
\left(\mathrm{d}^{\prime \prime} \varphi, \psi\right)=\left(\varphi, \delta^{\prime \prime} \psi\right), \quad\left(\mathrm{d}^{\prime} \varphi, \psi\right)=\left(\varphi, \delta^{\prime} \psi\right),
$$

c'est-à-dire que les opérateurs $\left(\mathrm{d}^{\prime \prime}, \delta^{\prime \prime}\right)$ et $\left(\mathrm{d}^{\prime}, \delta^{\prime}\right)$ sont adjoints par rapport au produit scalaire (3.15). Comme $\mathrm{d}$ et $\delta$ sont aussi adjoints il suit encore que $\partial$ et $\tilde{\partial}$ le sont aussi.

Nous pouvons alors définir l'opérateur de Laplace de l'opérateur d", qui est fondamental chez nous

$$
\Delta^{\prime \prime}=\mathrm{d}^{\prime \prime} \delta^{\prime \prime}+\delta^{\prime \prime} \mathrm{d}^{\prime \prime}
$$

et il s'ensuit que cet opérateur est du type $(0,0)$ et qu'il est autoadjoint.

Considérons maintenant la seconde connexion de la v.r.f. $V(\S 2.2)$ et convenons 
de noter par une barre la dérivée covariante par rapport à celle-ci. Alors, il suit de (2.21) et (3.5)

$$
\begin{aligned}
& \mathrm{d}^{\prime \prime} \varphi=\frac{(-1)^{p}}{p ! q !(q+1) !} \varepsilon_{u_{1} \ldots u_{q}+1}^{v v_{1} \ldots v_{q}} \varphi_{a_{1} \ldots a_{p} v_{1} \ldots v_{q} / v} \\
& . \mathrm{d} x^{a_{1}} \wedge \ldots \wedge \mathrm{d} x^{a_{p}} \wedge \theta^{u_{1}} \wedge \ldots \wedge \theta^{u_{q}+1}
\end{aligned}
$$

ce qui est une expression invariante de l'opérateur $\mathrm{d}^{\prime \prime}$.

Supposons encore, pour simplicité, que $V$ est un espace de Reinhart. Alors, on a d'abord

$$
\tilde{h}^{a b}{ }_{1 v}=0
$$

et puis

$$
\begin{gathered}
\eta_{1 \ldots n+m / v}=\frac{\partial \eta_{1 \ldots n+m}}{\partial x^{v}}-\sum_{i=1}^{n+m} \Gamma_{i v}^{h} \eta_{1 \ldots, i-1, h, i+1, \ldots, n+m}= \\
=\frac{\partial \sqrt{ }\left(h_{1} h_{2}\right)}{\partial x^{v}}-\sqrt{ }\left(h_{1} h_{2}\right) \sum_{u=n+1}^{n+m} \Gamma_{u v}^{u}=\frac{\partial \sqrt{ }\left(h_{1} h_{2}\right)}{\partial x^{v}}-\sqrt{ }\left(h_{1} h_{2}\right) \frac{\partial \log \sqrt{ } h_{2}}{\partial x^{v}}=\sqrt{ } h_{2} \frac{\partial \sqrt{ } h_{1}}{\partial x^{v}},
\end{gathered}
$$

d'où, compte tenu de ce que pour un espace de Reinhart $\partial h_{1} / \partial x^{v}=0$, il suit

$$
\eta_{1 \ldots n+m / v}=0 \text {. }
$$

On a aussi la relation, valable en général, $\tilde{h}_{/ w}^{u v}=0$.

Les formules établies ci-dessus permettent d'appliquer le calcul classique de [5], qui donne l'opérateur $\delta$, et d'obtenir l'expression invariante de l'opérateur $\delta^{\prime \prime}$

$$
\begin{gathered}
\delta^{\prime \prime} \varphi=\frac{(-1)^{p-1}}{(n-p) ! p !(q-1) !} \tilde{h}^{u v} \varphi_{a_{1} \ldots a_{p} v v_{1} \ldots v_{q-1} / u} \\
. \mathrm{d} x^{a_{1}} \wedge \ldots \wedge \mathrm{d} x^{a_{p}} \wedge \theta^{v_{1}} \wedge \ldots \wedge \theta^{v_{q-1}} .
\end{gathered}
$$

Il est à remarquer que, dans le cas d'une v.r.f. quelconque, la formule de $\delta^{\prime \prime}$ a encore d'autres termes, mais ceux-ci ne contiennent pas les dérivées des $\varphi$.

Maintenant, nous appliquerons les formules (3.20), (3.21) et (3.24) pour trouver l'expression de l'opérateur $\Delta^{\prime \prime}$. Nous trouvons d'abord pour les composantes de la form $\Delta^{\prime \prime} \varphi$

$$
\begin{gathered}
\left(\Delta^{\prime \prime} \varphi\right)_{a_{1} \ldots a_{p} u_{1} \ldots u_{q}}=-\frac{1}{n-p} \tilde{h}^{u v} \varphi_{a_{1} \ldots a_{p} u_{1} \ldots u_{q} / v u}+ \\
+\frac{1}{(n-p) !(q-1) !} \varepsilon_{u_{1} \ldots u_{q}}^{w v_{2} \ldots v_{q}-1} \tilde{h}^{u v}\left(\varphi_{a_{1} \ldots a_{p} v v_{1} \ldots v_{q-1} / w u}-\varphi_{a_{1} \ldots a_{p} v v_{1} \ldots v_{q-1} / u w}\right)
\end{gathered}
$$


et puis, en tenant compte des formules de commutation des dérivés covariantes [3] et des formules $(2.25),(2.26)$

$$
\begin{gathered}
\left(\Delta^{\prime \prime} \varphi\right)_{a_{1} \ldots a_{p} u_{1} \ldots u_{q}}=-\frac{1}{(n-p) !} \tilde{h}^{u v} \varphi_{a_{1} \ldots a_{p} u_{1} \ldots u_{q} / v u}+ \\
+\frac{1}{(n-p) !} \sum_{h=1}^{q} R_{u_{h}}^{t} \varphi_{a_{1} \ldots a_{p} u_{1} \ldots t \ldots u_{q}}+\frac{1}{2(n-p) !} \sum_{s=1}^{q} \sum_{h=1}^{q} R^{v u}{ }_{u_{s} u_{h}} \varphi_{a_{1} \ldots a_{p} u_{1} \ldots u \ldots v \ldots u_{q}} .
\end{gathered}
$$

Bien entendu, cette formule est valable pour les espaces de Reinhart. Dans le cas général, il y a encore d'aures termes dans le second membre de (3.25), mais ces termes ne contiennent pas les secondes dérivées des fonctions $\varphi$.

Dans la théorie classique des formes harmoniques, l'opérateur de Laplace est strictement elliptique, d'où il résulte quelques résultats très forts. Dans notre théorie, on voit de (3.25) que $\Delta^{\prime \prime}$ n'est pas strictement elliptique et il y a des exemples, dus à Reinhart [7], qui montrent que les résultats mentionnés ci-dessus ne sont plus valables en général. Pourtant, on peut obtenir des résultats moins forts mais assez importants.

Une forme différentielle $\varphi$ sur $V$ sera nommée $\Phi$-harmonique si elle vérifie la condition

$$
\Delta^{\prime \prime} \varphi=0 \text {. }
$$

Comme pour les formes harmoniques [5], on voit que, $V$ étant compacte, l'équation (3.26) est équivalente au système

$$
\mathrm{d}^{\prime \prime} \varphi=\delta^{\prime \prime} \varphi=0 .
$$

Il est clair que les formes $\Phi$-harmoniques du type $(p, q)$ forment un espace linéaire réel et celui-ci sera noté par $\mathscr{H}^{p q}(V)$. Évidemment, si $\varphi$ est une forme harmonique au sens classique $(\mathrm{d} \varphi=\delta \varphi=0)$ et si elle est du type $(p, q)$, elle vérifie aussi (3.27) et est donc $\Phi$-harmonique. Il s'en ensuit que, si l'on note par $H^{p q}(V)$ l'espace des formes harmoniques du type $(p, q)$ sur $V$, celui-ci est un sous-espace de $\mathscr{H}^{p q}(V)$.

De (3.27) il suit qu'une forme $\Phi$-harmonique du type $(p, q)$ est $\mathrm{d}^{\prime \prime}$-fermée, donc, selon le théorème 3.2, elle définit une classe de cohomologie de $H^{q}\left(V, \Phi^{p}\right)$. Démontrons que si dans une classe de $H^{q}\left(V, \Phi^{p}\right)$ il y a une forme $\Phi$-harmonique, elle est unique. En effet, si $\varphi$ et $\psi$ sont deux formes $\Phi$-harmoniques telles que

$$
\varphi-\psi=\mathrm{d}^{\prime \prime} \lambda,
$$

alors $\mathrm{d}^{\prime \prime} \lambda$ est aussi $\Phi$-harmonique et nous avons

$$
\left(\mathrm{d}^{\prime \prime} \lambda, \mathrm{d}^{\prime \prime} \lambda\right)=\left(\lambda, \delta^{\prime \prime} \mathrm{d}^{\prime \prime} \lambda\right)=0
$$

d'où $\mathrm{d}^{\prime \prime} \lambda=0$ et $\varphi=\psi$.

Il suit évidemment que $\mathscr{H}^{p q}$ est isomorphe à un sous-espace de $H^{q}\left(V, \Phi^{p}\right)$ et nous avons démontré le 
Théorème 3.3. Pour une v.r.f. compacte et orientée $V, \mathscr{H}^{p q}(V)$ est, à un isomorphisme près, un sous-espace de $H^{q}\left(V, \Phi^{p}\right)$ et $H^{p q}(V)$ est un sous-espace de $\mathscr{H}^{p q}(V)$.

Ce théorème est valable sans la condition que $V$ soit un espace de Reinhart, car les considérations qui interviennent dans sa démonstration le sont également.

Nous dirons qu'une v.r.f. compact et orientée $V$ est cohomologiquement régulière si, pour tout $p$ et $q$, elle vérifie la condition $H^{q}\left(V, \Phi^{p}\right)=\mathscr{H}^{p q}(V)$.

3.3. Nous allons déduire maintenant quelques conséquences du théorème (3.3).

Commençons par le résultat simple suivant

Théorème 3.4. Pour toute v.r.f. compacte et orientée V, on a

$$
\operatorname{dim} H^{m}(V, \Phi) \geqq 1 .
$$

En effet, considérons la forme du type $(0, m)$

$$
\tau=\sqrt{ }\left(h_{2}\right) \theta^{n+1} \wedge \ldots \wedge \theta^{n+m},
$$

qui est (on le voit aisément) globalement définie sur $V$. Elle est d"-fermée et, avec (3.13), on trouve

$$
* \tau=(-1)^{n m} \sqrt{ }\left(h_{1}\right) \mathrm{d} x^{1} \wedge \ldots \wedge \mathrm{d} x^{n} .
$$

Faisons maintenant une transformation conforme de la métrique $g$ de $V$, telle que pour la nouvelle métrique on ait $h_{1}=1$. Une pareille transformation est toujours possible et nous pouvons considérer l'harmonicité par rapport à cette nouvelle métrique.

Alors, il suit

$$
\mathrm{d}^{\prime \prime} * \tau=0,
$$

donc $\delta^{\prime \prime} \tau=0$, ce qui montre que $\tau$ est une forme $\Phi$-harmonique non-nulle et, compte tenu du théorème 3.3, le résultat annoncé en résulte. (Dans [13], ce résultat a été donné seulement pour le cas particulier d'un espace de Reinhart; dans ce cas la transformation conforme de ci-dessus n'est plus nécessaire.)

On peut aussi obtenir des résultats corresponclants au théorème classique de Bochner-Lichnerowicz et ceux-ci seront démontrés dans la manière de [3]. Ainsi nous avons le

Théorème 3.5. Soit V une v.r.f. compacte et orientée et supposons que le tenseur de Ricci $\varrho_{i j}$ de la première connexion de $V$ vérifie les conditions: a) $\varrho_{a}=0$, b) $\varrho_{a b} \xi^{a} \xi^{b}$ est une forme positivement définie. Alors on a

$$
\operatorname{dim} H^{1}(V, \Phi) \geqq b^{1},
$$

où $b^{1}$ est le premier nombre de Betti de la variété $V$. 
Compte tenu du théorème (3.3), il est évident que, pour démontrer cette affirmation, il suffit de montrer que, dans nos hypothèses, toute forme harmonique du premier degré est nécessairement une forme du type $(0,1)$.

Soit

$$
\varphi=\varphi_{a} \mathrm{~d} x^{a}+\varphi_{u} \theta^{u}
$$

une forme de Pfaff. $\Delta \varphi$, où $\Delta$ est l'opérateur classique de Laplace, peut être calculé à l'aide de la formule connue (voir par exemple [3]); c'est la somme d'une forme du type $(1,0)$ et d'une forme du type $(0,1)$ et, en notant par le symbole ,;" "la dérivée covariante par rapport à la première connexion de $V$, la partie du type $(1,0)$ de $\Delta \varphi$ a les composantes (on tiendra aussi compte de l'hypothèse a))

$$
(\Delta \varphi)_{c}=-\tilde{h}^{a b} \varphi_{c ; a b}-\tilde{h}^{u v} \varphi_{c ; u v}+\varrho_{c}^{a} \varphi_{a} .
$$

Si $\varphi$ est une forme harmonique, $(\Delta \varphi)_{c}=0$, et nous pouvons écrire la relation

$$
\int_{V}(\Delta \varphi)_{c} \varphi^{c} \mathrm{~d} V=0
$$

ou, d'après (3.30)

$$
\int_{V} \varrho_{a c} \varphi^{a} \varphi^{c} \mathrm{~d} V-\int_{V} \tilde{h}^{a b} \varphi_{c ; a b} \varphi^{c} \mathrm{~d} V-\int_{V} \tilde{h}^{u v} \varphi_{c ; u v} \varphi^{c} \mathrm{~d} V=0 .
$$

Considérons maintenant la fonction $\lambda=\varphi_{c} \varphi^{c}$. Nous avons

$$
(\delta \mathrm{d} \lambda, 1)=(\mathrm{d} \lambda, \mathrm{d} 1)=0
$$

c'est-à-dire

$$
\int_{V}\left(\tilde{h}^{a b} \lambda_{; a b}+\tilde{h}^{u v} \lambda_{; u v}\right) \mathrm{d} V=0 .
$$

D'ici, en explicitant les dérivées qui interviennent, on peut calculer la somme des deux dernier termes de (3.32) et on trouve

$$
\int_{V} \varrho_{a b} \varphi^{a} \varphi^{b} \mathrm{~d} V+\int_{V}\left(\tilde{h}^{a b} \varphi_{c ; a} \varphi_{; b}^{c}+\tilde{h}^{u v} \varphi_{c ; u} \varphi_{; v}^{c}\right) \mathrm{d} V=0 .
$$

L'hypothèse b) de notre théorème montre que les deux termes de (3.33) sont nonnégatifs, donc

$$
\varrho_{a b} \varphi^{a} \varphi^{b}=0
$$

et, en appliquant de nouveau l'hypothèse b), $\varphi^{a}=0$ c.q.f.d.

D'une manière analogue on peut obtenir le 
Théorème 3.6. Soit $V$ un espace de Reinhart, compact et orienté et soit $R_{i j}$ le tenseur de Ricci de la seconde connexion de V. Si la forme $R_{u v} \xi^{u} \xi^{v}$ est positivement définie, il n'y a pas sur $V$ de formes $\Phi$-harmoniques du type $(0,1)$ non nulles.

En effet, avec la formule (3.25), on a pour une forme $\varphi$ du type $(0,1)$

$$
\left(\Delta^{\prime \prime} \varphi, \varphi\right)=\frac{1}{n !} \int_{V}\left(-\tilde{h}^{v w} \varphi_{u / w v} \varphi^{u}+R_{u}^{t} \varphi_{t} \varphi^{u}\right) \mathrm{d} V .
$$

Considérons d'autre par la fonction $\lambda=\varphi_{u} \varphi^{u}$. On a alors

$$
\left(\delta^{\prime \prime} d^{\prime \prime} \lambda, 1\right)=\left(d^{\prime \prime} \lambda, d^{\prime \prime} 1\right)=0,
$$

d'où

$$
\int_{V} \tilde{h}^{u w} \lambda_{/ w v} \mathrm{~d} V=0
$$

En explicitant ici les dérivées covariantes, on obtient la suivante forme transformée de (3.34)

$$
\left(\Delta^{\prime \prime} \varphi, \varphi\right)=\frac{1}{n !} \int_{V}\left(\tilde{h}^{v w} \varphi_{u / w} \varphi_{/ v}^{u}+R_{u t} \varphi^{u} \varphi^{t}\right) \mathrm{d} V .
$$

Si $\varphi$ est $\phi$-harmonique, $\Delta^{\prime \prime} \varphi=0$ et (3.55) donne le résultat désiré par le même raisonnemenet qu'à la fin de la démonstration du théorème 3.5.

La formule (3.35) montre encore que, pour un espace de Reinhart quelconque, si $R_{u t} \varphi^{u} \varphi^{t} \geqq 0$, la forme $\varphi$ est $\Phi$-harmonique si et seulement si $\varphi_{u / t}=0$ et $R_{u t} \varphi^{u} \varphi^{t}=0$.

$\mathrm{Si}$ aux hypothèses du théorème 3.6 nous ajoutons l'hypothèse que $V$ soit cohomologiquement régulière, il résulte $H^{1}(V, \Phi)=0$.

Pour obtenir encore un résultat, rappelons qu'au $\S 2.3$ nous avons considéré les faisceaux $\Xi$ et $\Lambda$ et que nous avons démontré la formule (2.33). Un germe du faisceau $\Lambda$, c'est-à-dire un germe de section feuilletée du fibre $D^{\perp}(V)$ est représenté à l'aide des quantités $\xi^{a}$ du $\S 2.3$ et nous pouvons lui associer le germe de forme différentielle du type $(1,0)$ défini par les composantes $h_{a b} \xi^{b}$. Dans le cas d'un espace de Reinhart, ce dernier appartient évidemment au fásceau $\Phi^{1}$ et il est clair que nous obtenons ainsi un isomorphisme des faisceaux $\Lambda$ et $\Phi^{1}$, donc

$$
H^{q}(V, \Xi)=H^{q}(V, \Lambda)=H^{q}\left(V, \Phi^{1}\right) \quad(q \geqq 1)
$$

et ces groupes peuvent se calculer à l'aide du théorème 3.2.

On sait, d'après Kodaira et SPENCER [4], que le groupe $H^{1}(V, \Xi)$ a une importance spéciale, car il contient les déformations infinitésimales de la structure feuilletée de $V$; cette structure est donc rigide (n'a pas de déformations) si $H^{1}(V, \Xi)=0$.

Alors, la formule (3.36) et les méthodes considérées auparavant nous permettent d'établir le 
Théorème 3.7. Soit $V$ un espace de Reinhart, compact, orienté et cohomologiquement régulier et soit $R_{i j}$ le tenseur de Ricci de la seconde connexion de V. Alors, si la forme $R_{u v} \xi^{u} \xi^{v}$ est positivemenet définie, la structure feuilletée de V est rigide.

En effet, selon les hypothèses et les considérations de ci-dessus, il suffit de montrer qu'on a $\mathscr{H}^{11}(V)=0$.

Soit

$$
\alpha=\alpha_{a u} \mathrm{~d} x^{a} \wedge \theta^{u}
$$

une forme du type (1.1) sur $V$. En procédant exactement comme pour le théorème 3.6, on obtient

$$
\left(\Delta^{\prime \prime} \alpha, \alpha\right)=\frac{1}{(n-1) !} \int_{V}\left(\tilde{h}^{u w} \alpha_{a u / v} \alpha_{/ w}^{a u}+R_{u v} \alpha_{a}^{u} \alpha^{a v}\right) \mathrm{d} V,
$$

d'où suit l'affirmation du théorème.

Pour le cas d'une structure feuilletée générale, on donne [4] une résolution du faisceau $\Xi$ à l'aide des formes-jet. Mais il est à remarquer qu'on peut obtenir un résultat plus simple, en cherchant une résolution pour le faisceau $\Lambda$.

En effet, considérons les formes différentielles sur $V$ à valeurs dans le fibré transversal $D^{\perp}(V)$. La notion de type est valable pour ces formes aussi et une forme du type $(p, q)$ a les composantes locales

$$
\xi^{a}=\frac{1}{p ! q !} \xi_{b_{1} \ldots b_{p} u_{1} \ldots u_{q}}^{a} \mathrm{~d} x^{b_{1}} \wedge \ldots \wedge \mathrm{d} x^{b_{p}} \wedge \theta^{u_{1}} \wedge \ldots \wedge \theta^{u_{q}} .
$$

Il est aisé de voir que les formes $\mathrm{d}^{\prime \prime} \xi^{a}$ sont alors les composantes d'une autre forme à valeurs dans $D^{\perp}(V)$, car on a, compte tenu de (2.18) et (3.21),

$$
\begin{gathered}
\mathrm{d}^{\prime \prime} \xi^{a}=\frac{(-1)^{p}}{p ! q !(q+1) !} \varepsilon_{u_{1} \ldots u_{q}+\xi^{2}}^{v v_{1} \ldots v_{q}} \xi_{b_{1} \ldots b_{p} v_{1} \ldots v_{q} / v}^{a} . \\
. \mathrm{d} x^{b_{1}} \wedge \ldots \wedge \mathrm{d} x^{b_{p}} \wedge \theta^{u_{1}} \wedge \ldots \theta^{u_{q+1}} .
\end{gathered}
$$

Donc, $\mathrm{d}^{\prime \prime}$ définit un opérateur qui sera noté aussi par $\mathrm{d}^{\prime \prime}$, sur les formes à valeurs dans $D^{\perp}(V)$ et, évidemment, le théorème 3.1 reste valable.

Alors, si nous notons par $\mathscr{E}^{p q}$ le faisceau des germes de formes du type $(p, q)$ et à valeurs dans $D^{\perp}(V)$ nous trouvons que

$$
0 \rightarrow \Lambda \stackrel{i}{\rightarrow} \mathscr{E}^{00} \stackrel{\mathrm{d}^{\prime \prime}}{\rightarrow} \mathscr{E}^{01} \stackrel{\mathrm{d}^{\prime \prime}}{\rightarrow} \ldots \stackrel{\mathrm{d}^{\prime \prime}}{\rightarrow} \mathscr{E} 0 \mathrm{Em} \rightarrow 0,
$$

où $i$ est l'injection naturelle, est une résolution fine de $\Lambda$.

Nous avons donc le

Théorème 3.8. $H^{q}(V, \Xi)=H^{q}(V, \Lambda)=F^{0 q} / \mathrm{d}^{\prime \prime} E^{0, q-1}$, où $E^{p q}$ est l'espace des formes à valeurs dans $D^{\perp}(V)$ et du type $(p, q)$ et $F^{p q}$ le sous-espace du précédent formé par les formes $\mathrm{d}^{\prime \prime}$-fermées. 
Donc, dans l'étude de déformations des structures feuilletées on peut utiliser la résolution (3.40) au lieu de celle de [4]; mais cette dernière a l'avantage de s'appliquer dans le cas d'une structure multifeuilletée quelconque.

\section{CHAMPS FEUILLETÉS DE VECTEURS DE KILLING}

4.1. Il est bien connu que les méthodes du $\S 3.3$ sont aussi appliquables à l'étude de l'existence des champs de vecteurs de Killing dans un espace de Riemann [3]. Dans le cas d'une v.r.f. il est naturel d'envisager, par ces méthodes, le problème de l'existence des transformations infinitésimales conservant, en même temps, la structure feuilletée et la métrique et c'est justemement ce que nous allons faire ici. Nous employons les mêmes notations qu'aux paragraphes précédents; rappelons aussi que toutes les composantes des tenseurs sont prises par rapport aux bases $\left(X_{a}, X_{u}\right)$.

Soit $\xi$ un champ de vecteurs, définissant une transformation infinitésimale de la v.r.f. $V$. Cette transformation conserve la structure feuilletée de $V$ si et seulement is

$$
L_{\xi} X_{u}=a_{u}^{v} X_{v},
$$

où $L_{\xi}$ est la dérivée de Lie par rapport au champ $\xi$ et $a_{a}^{v}$ sont des fonctions régulières définies dans les voisinages des points où l'on considère la relation (4.1).

En vue d'expliciter (4.1), considérons d'abord les formules qu'on établit par des calculs directs

$$
\left[X_{u}, X_{v}\right]=0, \quad\left[X_{a}, X_{u}\right]=\frac{\partial}{\partial x^{u}} t_{a}^{v} X_{v}, \quad\left[X_{a}, X_{b}\right]=\tau_{a b}^{u} X_{u},
$$

où $t_{a}^{v}$ sont donnés par la formule (2.9) et

$$
\tau_{a b}^{u}=\frac{\partial}{\partial x^{b}} t_{a}^{u}-\frac{\partial}{\partial x^{a}} t_{b}^{u}+t_{a}^{v} \frac{\partial}{\partial x^{v}} t_{b}^{u}-t_{b}^{v} \frac{\partial}{\partial x^{v}} t_{a}^{u} .
$$

(Rappelons que $\tau_{a b}^{u}=0$ est la condition d'intégrabilité de la distribution transversale $\mathscr{D}^{\perp}$.)

Puis, notons

$$
\xi=\xi^{a} X_{a}+\xi^{u} X_{u} .
$$

Alors, la condition (4.1), qui est justemenet

$$
\left[\xi, X_{u}\right]=a_{u}^{v} X_{v},
$$

donne

$$
\frac{\partial \xi^{a}}{\partial x^{u}}=0
$$


Donc, selon la formule (2.30), la transformation infinitésimale $\xi$ conserve la structure feuilletée de $V$ si et seulement si le champ $\xi$ est feuilleté.

Il est important de remarquer que, compte tenu de (2.18), la condition (4.6) est équivalente à

$$
\xi_{/ u}^{a}=0,
$$

où la barre désigne la dérivée covariante par rapport à la seconde connexion de $V$.

Il est aussi à remarquer que les champs de vecteurs appartenant à la distribution structurelle $\mathscr{D}\left(\xi^{a}=0\right)$ définissent des transformations infinitésimales qui conservent la structure feuilletée.

Quant à la métrique, elle est conservée par $\xi$ si et seulement si ce dernier est un champ de Killing, c'est-à-dire

$$
L_{\xi} g=0 .
$$

On sait bien (voir par exemple [3]), qu'à l'aide de la première connexion de $V$, la condition (4.8) revient à

$$
\xi_{i ; j}+\xi_{j ; i}=0 .
$$

Pour expliciter la condition (4.8), à l'aide de la seconde connexion de $V$, nous partons de la formule (voir par exemple [12])

$$
\left(L_{\xi} g\right)(X, Y)=\xi g(X, Y)-g\left(L_{\xi} X, Y\right)-g\left(X, L_{\xi} Y\right)
$$

et, en tenant compte de (4.2), on arrive après des calculs aux conditions

$$
\begin{aligned}
& \left(L_{\xi} g\right)_{a b}=\xi_{a / b}+\xi_{b / a}+h_{a b / u} \xi^{u}=0, \\
& \left(L_{\xi} g\right)_{a u}=\left(L_{\xi} g\right)_{u a}=h_{a c} \xi_{/ u}^{c}+h_{u v} \xi_{/ a}^{v}-h_{u v} v_{c a}^{v} \xi^{c}=0, \\
& \left(L_{\xi} g\right)_{u v}=\xi_{u / v}+\xi_{v / u}+h_{u v / c} \xi^{c}=0 .
\end{aligned}
$$

Remarquons que, par suite de la formule (4.7), la seconde condition (4.11) revient à

$$
\xi_{/ a}^{v}-\tau_{c a}^{v} \xi^{c}=0,
$$

d'où, on trouve par un calcul simple

$$
\left[\xi, X_{a}\right]=b_{a}^{c} X_{c},
$$

avec certaines fonctions $b$. Ceci exprime le fait, géométriquement évident, que la transformation $\xi$ conserve aussi la distribution transverśale $\mathscr{D}^{\perp}$.

Donc, les transformations infinitésimales qui conservent en même temps la structure feuilletée et la métrique d'une v.r.f. sont celles définies par des champs feuilletés de vecteurs de Killing; ces derniers sont caractérisés par (4.6) et (4.9) ou par (4.7) et (4.11). 
4.2. Dorénavant, la variété $V$ sera de nouveau supposée une v.f.r. compacte et orientée. Alors, selon un résultat classique [3], si la courbure de Ricci de la première connexion de $V$ définit une forme quadratique négativement définie, il n'y a pas sur $V$ de champs de vecteurs de Killing, donc il n'y a pas de champs feuilletés de Killing non plus. On voit ainsi que, pour obtenir des résultats plus spécifiques, il faut employer la seconde connexion de la variété.

Occupons-nous d'abord des champs de vecteurs $\xi$, qui appartiennent à la distribution structurelle $\mathscr{D}$; ils sont feuilletés, comme nous l'avons déjà remarqué. Nous avons $\xi^{c}=0$, et la dernière des conditions (4.11) revient à

$$
\xi_{u / v}+\xi_{v / u}=0 \text {. }
$$

Supposons, de plus, que $V$ est un espace de Reinhart. Alors on a pour la forme $\xi=\xi_{u} \theta^{u}$

$$
\delta^{\prime \prime} \xi=-\frac{1}{n !} \tilde{h}^{u v} \xi_{v / u}
$$

donc, si (4.14) est vérifiée,

$$
\delta^{\prime \prime} \xi=0 .
$$

Mais alors, par suite de la définition de l'opérateur $\Delta^{\prime \prime}, \xi$ vérifie nécessairement la condition

$$
\left(\Delta^{\prime \prime} \xi, \xi\right)-\left(d^{\prime \prime} \xi, d^{\prime \prime} \xi\right)=0
$$

Avec (4.14), on trouve

$$
\mathrm{d}^{\prime \prime} \xi=-\xi_{u / v} \theta^{u} \wedge \theta^{v}
$$

ce qui donne

$$
\left(\mathrm{d}^{\prime \prime} \xi, \mathrm{d}^{\prime \prime} \xi\right)=2 \int_{V} \tilde{h}^{v w} \xi_{u / w} \xi_{/ v}^{u} \mathrm{~d} V .
$$

Quant au produit $\left(\Delta^{\prime \prime} \xi, \xi\right)$ il est donné par la formule (3.35) si l'on y remplace $\varphi$ par $\xi$.

En introduisant les résultats dans (4.16), nous obtenons

$$
\frac{1}{n !} \int_{V} R_{u t} \xi^{t} \xi^{u} \mathrm{~d} V-\left(2-\frac{1}{n !}\right) \int_{V} \tilde{h}^{v w} \xi_{u / w} \xi^{u}{ }^{\prime} \mathrm{d} V=0
$$

Alors, en remarquant que la seconde partie du premier membre de la formule (4.19) est non-positive, il suit, comme au $\S 3.3$, le

Théorème 4.1. Soit $V$ un espace de Reinhart compact et orienté et $R_{i j}$ le tenseur de Ricci de la seconde connexion de $V$. Alors, si la forme $R_{u v} \xi^{u} \xi^{v}$ est négativement 
définie, il n'y a pas sur $V$ de champs de vecteurs de Killing appartenant à la distribution structurelle $\mathscr{D}$.

Théorème 4.2. Soit $V$ un espace de Reinhart compact et orienté et soit $\xi$ un champ de vecteurs de Killing, appartenant à $\mathscr{D}$. Alors, si $R_{u v} \xi^{u} \xi^{v} \leqq 0$, on a $R_{u v} \xi^{u} \xi^{v}=0$ et $\xi_{/ v}^{u}=0$.

Remarquons d'ailleurs que nous avons démontré des résultats plus forts que les énoncés des théorèmes 4.1 et 4.2 , car nous avons employé seulement la dernière des conditions (4.11); en effet les théorèmes 4.1 et 4.2 restent valables si l'on remplace la condition que le champ $\xi$ soit un champ de Killing par la condition que la transformation infinitésimale $\xi$ conserve la métrique du fibré structurel $D(V)$.

Pour obtenir un résultat sur les champs feuilletés de Killing généraux, introduisons aussi les tenseurs

$$
B_{i j}=R_{h i j}^{h}, \quad C_{i j}=h_{u v / i} \tilde{h}_{/ j}^{u v} .
$$

Nous démontrerons alors le

Théorème 4.3. Soit $V$ un espace de Reinhart compact et orienté, qui vérifie les conditions: a) $B_{u a}=0$, b) la forme $R_{u v} \xi^{u} \xi^{v}$ est négativement définie, c) la forme $C_{a b} \xi^{a} \xi^{b}$ est positivement définie. Alors, il n'y a pas sur $V$ de champs feuilletés de vecteurs de Killing, non nuls.

Soient $\left(\xi^{a}, \xi^{u}\right)$ les composantes du champ feuilleté de Killing $\xi$. Avec la dernière des conditions (4.11), on trouve alors pour la forme $\zeta=\xi_{u} \theta^{u}$

$$
\delta^{\prime \prime} \zeta=\frac{1}{2 n !} \tilde{h}^{u v} h_{u v / c} \xi^{c}
$$

et puis, en vue de (4.7) et de la relation évidente $h_{u v / t}=0$

$$
\mathrm{d}^{\prime \prime} \delta^{\prime \prime} \zeta=\frac{1}{2 n !} \tilde{h}^{u v} h_{u v / c t} \xi^{c} \theta^{t}
$$

Il suit alors

$$
\left(\delta^{\prime \prime} \zeta, \delta^{\prime \prime} \zeta\right)=\left(\zeta, \mathrm{d}^{\prime \prime} \delta^{\prime \prime} \zeta\right)=\frac{1}{2 n !} \int_{V} \tilde{h}^{u v} h_{u v / c t} \xi^{c} \xi^{t} \mathrm{~d} V
$$

Mais, en employant de nouveau $h_{u v / t}=0$ et en tenant compte des formules classiques de commutation et des formules (2.25), nous trouvons

donc

$$
h_{u v / c t}=h_{u v / c t}-h_{u v / t c}=R_{v u t c}+R_{u v t c},
$$

$$
\tilde{h}^{u v} h_{u v / c t}=2 B_{t c}
$$


et (4.22) devient

$$
\left(\delta^{\prime \prime} \zeta, \delta^{\prime \prime} \zeta\right)=\frac{1}{n !} \int_{V} B_{u a} \xi^{u} \xi^{a} \mathrm{~d} V
$$

De l'hypothèse a) il suit alors $\left(\delta^{\prime \prime} \zeta, \delta^{\prime \prime} \zeta\right)=0$, c'est-à-dire $\delta^{\prime \prime} \zeta=0$.

Alors, c'est maintenant la forme $\zeta$ qui vérifie la condition (4.16)

$$
\left(\Delta^{\prime \prime} \zeta, \zeta\right)-\left(\mathrm{d}^{\prime \prime} \zeta, \mathrm{d}^{\prime \prime} \zeta\right)=0
$$

Ici, le premier terme est donné, de nouveau, par (3.35) et pour le second on trouve, en employant de nouveau (4.11)

$$
\left(\mathrm{d}^{\prime \prime} \zeta, \mathrm{d}^{\prime \prime} \zeta\right)=2 \int_{V} \tilde{h}^{w w} \xi_{u / w} \xi^{u}{ }_{1 v} \mathrm{~d} V+\frac{1}{2} \int_{V} C_{a b} \xi^{a} \xi^{b} \mathrm{~d} V .
$$

Il suit que le champ $\xi$ vérifie nécessairement la condition

$$
\frac{1}{n !} \int_{V} R_{u v} \xi^{u} \xi^{v} \mathrm{~d} V-\left(2-\frac{1}{n !}\right) \int_{V} \tilde{h}^{v w} \xi_{u / w} \xi^{u}{ }_{l v} \mathrm{~d} V-\frac{1}{2} \int_{V} C_{a b} \xi^{a} \xi^{b} \mathrm{~d} V=0
$$

d'où on obtient, de la manière déjà connue, la conclusion du théorème.

Il y a aussi un analogue du théorème 4.2.

Théorème 4.4. Soit $V$ un espace de Reinhart compact et orienté et qui vérifie la condition $B_{u a}=0$. Soit encore $\xi$ un champ feuilleté de vecteurs de Killing sur $V$. Alors, les conditions

$$
R_{u v} \xi^{u} \xi^{v} \leqq 0, \quad C_{a b} \xi^{a} \xi^{b} \geqq 0
$$

impliquent les relations

$$
R_{u v} \xi^{u} \xi^{v}=0, \quad C_{a b} \xi^{a} \xi^{b}=0, \quad \xi_{/ v}^{u}=0 .
$$

Enfin, remarquons, pour terminer, une conséquence simple des conditions (4.7) et (4.11). Si $\xi$ est un champ feuilleté de Killing d'une v.r.f. $V$ quelconque (sans conditions de compacité), appartenant à la distribution transver:ale $\mathscr{D}^{\perp}$ et si parmi les équations

$$
h_{u v / c} \xi^{c}=0, \quad \tau_{c a}^{v} \xi^{c}=0,
$$

il y en a $n$ linéairement indépendantes, alors $\xi=0$.

\section{Bibliographie}

[1] Cartan E.: Leçons sur la géométrie des espaces de Riemann, Gauthier-Villars, Paris, 1946.

[2] Chern S. S.: Complex manifolds without potential theory. D. Van Nostrand Comp. Princeton, 1967. 
[3] Goldberg S. I.: Curvature and Homology, Academic Press, New York, 1962.

[4] Kodaira K., Spencer D. C.: Multifoliate structures. Ann. of Math. 74, 1961, p. $52-100$.

[5] Lichnerowicz A.: Théorie globale des connexions et des groupes d'holonomie. Ed. Cremonese, Roma, 1955.

[6] Miron R., Papuc D. I.: Sur la théorie locale des distributions définies sur un espace à connexion affine. Rev. Roumaine de Math. pures et appl., XII, 1967, p. 537-543.

[7] Reinhart B. L.: Harmonic integrals on almost product manifolds. Trans. A.M.S. 88 (1958), p. $243-276$.

[8] Reinhart B. L.: Foliated manifolds with bundle-like metrics. Ann. of Math., 69, 1959, p. $119-132$.

[9] Reinhart B. L.: Harmonic integrals on foliated manifolds. Amer. M. J., 81, 1959, p. $529-536$.

[10] Sasaki $S .:$ On the differential geometry of tangent bundles of Riemannian manifolds, Tôhoku M. J., 10, 1958, p. 338-354.

[11] Spencer D. C.: De Rham theorems and Neumann decompositions associated with partial differential equations. "Structures feuilletées", Coll. C.N.R.S., Grenoble, 1963, p. 1-20.

[12] Vaisman I.: Sur quelques formules du calcul de Ricci global. Comm. Math. Helv., 41, $1966-67$, p. $73-87$.

[13] Vaisman I.: Sur la cohomologie des variétés riemanniennes feuilletées. C.R. Acad. Sc. Paris, t. 268, Série A, p. $720-723$.

Adresse de l'auteur: Université de Jassy, Chaire de Géométrie, Jassy, Roumanie. 